\title{
Gravitational waves from coalescing binaries: detection strategies and Monte Carlo estimation of parameters
}

\author{
R. Balasubramanian, B.S. Sathyaprakash, S. V. Dhurandhar \\ Inter-University Centre for Astronomy and Astrophysics, \\ Post Bag 4, Ganeshkhind, Pune 411 00\%, India
}

(June 13, 2018)

\begin{abstract}
The detection of gravitational waves from astrophysical sources is probably one of the most keenly awaited events in the history of astrophysics. The paucity of gravitational wave sources and the relative difficulty in detecting such waves, as compared to those in the electromagnetic domain, necessitate the development of optimal data analysis techniques to detect the signal, as well as to extract the maximum possible information from the detected signals. Coalescing binary systems are one of the most promising sources of gravitational waves. This is due to the fact that such sources are easier to model and thus one can design detection strategies particularly tuned to such signals. A lot of attention has been devoted in the literature studying such techniques and most of the work has revolved around the Weiner filtering and the maximum likelihood estimators of the parameters of the binary system. We investigate such techniques with the aid of differential geometry which provides geometric insight into the problem. Such a formalism allows to explore the merits and demerits of a detection scheme independent of the parameters chosen to represent the waveform. The formalism also generalises the problem of choosing an optimal set of templates to detect a known waveform buried in noisy data. We stress the need for finding a set of convenient parameters for the waveform and show that even after the inclusion of the second-order post-Newtonian corrections, the waveform can essentially be detected by employing a one-dimensional lattice of templates. This would be very useful both for the purpose of carrying out the simulations as well as for the actual detection process. After setting up such a formalism we carry out a Monte Carlo simulation of the detection process for the initial LIGO/VIRGO configuration for the first post-Newtonian corrected coalescing binary waveform. We compare the results of our simulations with the currently available estimates of the accuracies in the determination of the parameters and the probability distribution of the maximum likelihood estimators. Our results suggest that the covariance matrix underestimates, by over a factor of two, the actual errors in the estimation of parameters even when the signal-to-noise ratio is as high as 20. As only a tiny fraction of the events is expected to be detected with a signal-to-noise higher than this value, the covariance matrix is grossly inadequate to describe the errors in the measurement of the parameters of the waveform. It is found from our Monte Carlo simulations that the deviations from the covariance matrix are more in the case of the first post-Newtonian waveform than in the case of the Newtonian one. Inclusion of higher-order post-Newtonian corrections introduces new parameters that are correlated with those at the lower post-Newtonian waveform. Such correlations are expected to further increase the discrepancy of the covariance matrix results with those inferred from Monte Carlo simulations. Consequently, numerical simulations that take into account postNewtonian corrections beyond the first post-Newtonian order are needed in order to get a clearer picture about the accuracy in the determination of parameters. We find that with the aid of the instant of coalescence the direction to the source can determined more accurately than with the time of arrival.
\end{abstract}

\section{INTRODUCTION}

Laser interferometric detectors of gravitational waves such as the LIGO [1] and VIRGO [2] are expected to be operational by the turn of the century. Gravitational waves from coalescing binary systems of black holes and neutron stars are relatively 'clean' waveforms in the sense that they are easier to model and for this reason they are amongst the most important candidate sources for interferometric detectors. Binary systems are also valuable sources of astrophysical information as one can probe the universe up to cosmological distances. For instance, statistical analysis of several binary coalescences enables the estimation of the Hubble constant to an accuracy better than 10\% [3] [4]. Events that produce high signal-to-noise ratio can be potentially used to observe such non-linear effects, such as gravitational wave tails, and to put general relativity into test in the strongly non-linear 
regime [5]. Due to the weak coupling of gravitational radiation with matter the signal waveform is in general very weak and will not stand above the detector noise. In addition to the on-going efforts to reduce the noise, and hence increase the sensitivity of the detector, a considerable amount of research activity has gone into the development of efficient and robust data analysis techniques to extract signals buried in very noisy data. For a recent review on gravitational waves from compact objects and their detection see Thorne [6, ]6.

Various data analysis schemes have been suggested for the detection of the 'chirp' waveform from such systems [8 10]. Among them the technique of Weiner filtering is the most promising [10 12]. Briefly, this technique involves correlating the detector output with a set of templates, each of which is tuned to detect the signal with a particular set of parameters. This requires the signal to be known to a high level of accuracy. The fully general relativistic waveform from a coalescing binary system of stars is as yet unavailable. In the absence of such an exact solution, there have been efforts to find solutions perturbatively. Most of the work done in this area aims at computing the waveform correct to a high degree of accuracy so that theoretical templates computed based on them will obtain a large signal-to-noise ratio (SNR) when correlated with the detector output if the corresponding signal is present. In general, the number of parameters increases as we incorporate the higher order corrections. It is clear that the number of templates depends upon the number of signal parameters. As a consequence, the computing power for an on-line analysis will be greater for a larger number of parameters. In view of this restriction in computing power it is necessary to choose the templates in an optimal manner. This paper in part deals with this question. Investigations till now have been restricted to either choosing a finite subset of the signal space as templates 13, 14 or choosing templates from the 'Newtonian' or the 'first post-Newtonian' family of waveforms 15 18. We generalize this problem by using the language of differential geometry. We show that it is unnecessary to restrict oneself to templates that are matched exactly to any particular signal.

Differential geometry has been used in statistics before (see 19 and references therein) and the standard approach is to treat a set of parameterised probability distributions corresponding to a particular statistical model as a manifold. The parameters of the distribution serve as coordinates on this manifold. In statistical theory one frequently comes across the Fisher information matrix whose inverse gives a lower bound for the errors in the estimation of the parameters of a distribution. The Fisher information matrix turns out to be a very natural metric on the manifold of probability distributions and this metric can be used profitably in understanding the properties of a particular statistical model. Here in our paper we treat the set of coalescing binary signals corresponding to various parameters of the binary as a submanifold in the linear space of all detector outputs. We show in this paper that both the above mentioned manifolds are equivalent as far as their metrical properties are concerned. The geometric approach turns out to be useful not only in clarifying various aspects of signal analysis but also helps us to pose the question of optimal detection in a more general setting.

Once a signal has been detected we can estimate the parameters of the binary. We assume that the parameters of the signal are the same as those of the template with which the maximum correlation is obtained. The errors involved in such an estimation has been worked out by several authors [5, 18, 20, 27], for the case of 'high' SNR and for the Newtonian and post-Newtonian waveforms using a single and a network of detectors. For the case of low SNRs one has to resort to numerical simulations. We have started a project to carry out exhaustive numerical simulations specifically designed to compute the errors in the estimation of parameters and covariances among them at various post-Newtonian orders, for circular and eccentric orbits, with and without spin effects and for different optical configurations of the interferometer. In this paper we report the results for the case of the initial LIGO configuration taking into account only the first post-Newtonian corrections and assuming circular orbits. Going beyond this needs tremendous amount of computing power which is just becoming available.

The rest of the paper is organized as follows. In section 11 we describe the waveform from a coalescing binary system at various post-Newtonian orders. We introduce, following [28], a set of parameters called 'chirp times'. These parameters are found to be very convenient when we carry out Monte Carlo simulations. It turns out that the covariance matrix is independent of these parameters and hence it is sufficient to carry out the simulations only 
for a particular set of parameters. In section III we develop a geometric interpretation of signal analysis. We begin by introducing a metric on the manifold from a scalar product, which comes naturally from the theory of matched filtering and then show that this metric is the same as the one used by Amari 19]. Using the geometric approach we address the question of optimal filter placement and show that for the purpose of detection it is optimal to choose the templates outside the signal manifold. The covariance matrix of errors and covariances is shown to be the inverse of the metric on the manifold. In section IV we discuss the results of our simulations and compare the numerically obtained values and those suggested by the covariance matrix. We find substantial discrepancies in the predictions of the two methods. It is believed that the coalescing binary waveform shuts off abruptly at the onset of the plunge orbit. This has a major effect on the computations of the covariance matrix as well as on the Monte Carlo simulations. We discuss the effects of higher post-Newtonian corrections to the waveform. We also emphasisize the use of the instant of coalescence as a parameter in order to determine the direction to the source rather than the time of arrival [29]. Finally in section $\mathrm{V}$ we summarise our results and indicate future directions.

\section{COALESCING BINARY WAVEFORMS}

For the purpose of constructing templates for on-line detection, it is sufficient to work with the so called restricted post-Newtonian gravitational waveform. In this approximation the post-Newtonian corrections are incorporated only in the phase of the waveform, while ignoring corresponding corrections to the amplitude [30]. Consequently, the restricted postNewtonian waveforms only contain the dominant frequency equal to twice the orbital frequency of the binary computed up to the relevant order. In the restricted post-Newtonian approximation the gravitational waves from a binary system of stars, modeled as point masses orbiting about each other in a circular orbit, induce a strain $h(t)$ at the detector given by

$$
h(t)=A(\pi f(t))^{2 / 3} \cos [\varphi(t)],
$$

where $f(t)$ is the instantaneous gravitational wave frequency, the constant $A$ involves the distance to the binary, its reduced and total mass, and the antenna pattern of the detector [10], and the phase of the waveform $\varphi(t)$ contains several pieces corresponding to different post-Newtonian contributions which can be schematically written as

$$
\varphi(t)=\varphi_{0}(t)+\varphi_{1}(t)+\varphi_{1.5}(t)+\ldots
$$

Here $\varphi_{0}(t)$ is the dominant Newtonian part of the phase and $\varphi_{n}$ represents the $n$th order post-Newtonian correction to it. In the quadrupole approximation we include only the Newtonian part of the phase given by [10]

$$
\varphi(t)=\varphi_{0}(t)=\frac{16 \pi f_{a} \tau_{0}}{5}\left[1-\left(\frac{f}{f_{a}}\right)^{-5 / 3}\right]+\Phi,
$$

where $f(t)$ is the instantaneous Newtonian gravitational wave frequency given implicitly by

$$
t-t_{a}=\tau_{0}\left[1-\left(\frac{f}{f_{a}}\right)^{-8 / 3}\right]
$$

$\tau_{0}$ is a constant having dimensions of time given by

$$
\tau_{0}=\frac{5}{256} \mathcal{M}^{-5 / 3}\left(\pi f_{a}\right)^{-8 / 3},
$$

and $f_{a}$ and $\Phi$ are the instantaneous gravitational wave frequency and the phase of the signal, respectively, at $t=t_{a}$. The time elapsed starting from an epoch when the gravitational wave 
frequency is $f_{a}$ till the epoch when it becomes infinite will be referred to as the chirp time of the signal. In the quadrupole approximation $\tau_{0}$ is the chirp time. The Newtonian part of the phase is characterised by three parameters: (i) the time of arrival $t_{a}$ when the signal first becomes visible in the detector, (ii) the phase $\Phi$ of the signal at the time of arrival and (iii) the chirp mass $\mathcal{M}=\left(\mu^{3} M^{2}\right)^{1 / 5}$, where $\mu$ and $M$ are the reduced and the total mass of the binary, respectively. At this level of approximation two coalescing binary signals of the same chirp mass but of different sets of individual masses would be degenerate and thus exhibit exactly the same time evolution. This degeneracy is removed when post-Newtonian corrections are included.

When post-Newtonian corrections are included the parameter space of waveforms acquires an extra dimension. In this paper we show that even when post-Newtonian corrections up to relative order $c^{-4}$, where $c$ is the velocity of light, are included in the phase of the waveform it is possible to make a judicious choice of the parameters so that the parameter space essentially remains only three dimensional as far as the detection problem is concerned. It should, however, be noted that the evolution of the waveform must be known to a reasonably high degree of accuracy and that further off-line analysis would be necessary to extract useful astrophysical information.

With the inclusion of corrections up to second post-Newtonian order the phase of the waveform becomes 31]

$$
\varphi(t)=\varphi_{0}(t)+\varphi_{1}(t)+\varphi_{1.5}(t)+\varphi_{2}(t)
$$

where $\varphi_{0}(t)$ is given by (2.3) and the various post-Newtonian contributions are given by

$$
\begin{gathered}
\varphi_{1}(t)=4 \pi f_{a} \tau_{1}\left[1-\left(\frac{f}{f_{a}}\right)^{-1}\right] \\
\varphi_{1.5}(t)=-5 \pi f_{a} \tau_{1.5}\left[1-\left(\frac{f}{f_{a}}\right)^{-2 / 3}\right]
\end{gathered}
$$

and

$$
\varphi_{2}(t)=8 \pi f_{a} \tau_{2}\left[1-\left(\frac{f}{f_{a}}\right)^{-1 / 3}\right]
$$

Now $f(t)$ is the instantaneous gravitational wave frequency correct up to second postNewtonian order given implicitly by

$t-t_{a}=\tau_{0}\left[1-\left(\frac{f}{f_{a}}\right)^{-8 / 3}\right]+\tau_{1}\left[1-\left(\frac{f}{f_{a}}\right)^{-2}\right]-\tau_{1.5}\left[1-\left(\frac{f}{f_{a}}\right)^{-5 / 3}\right]+\tau_{2}\left[1-\left(\frac{f}{f_{a}}\right)^{-4 / 3}\right]$

In the above equations $\tau$ 's are constants having dimensions of time which depend only on the two masses of the stars and the lower frequency cutoff of the detector $f_{a}$. The total chirp time now consists of four pieces: the Newtonian contribution $\tau_{0}$ is given by (2.5) and the various post-Newtonian contributions are

$$
\begin{gathered}
\tau_{1}=\frac{5}{192 \mu\left(\pi f_{a}\right)^{2}}\left(\frac{743}{336}+\frac{11}{4} \eta\right), \\
\tau_{1.5}=\frac{1}{8 \mu}\left(\frac{m}{\pi^{2} f_{a}^{5}}\right)^{1 / 3}
\end{gathered}
$$

and 


$$
\tau_{2}=\frac{5}{128 \mu}\left(\frac{m}{\pi^{2} f_{a}^{2}}\right)^{2 / 3}\left(\frac{3058673}{1016064}+\frac{5429}{1008} \eta+\frac{617}{144} \eta^{2}\right)
$$

where $\eta=\mu / M$. The phase (2.6) contains the reduced mass $\mu$ in addition to the chirp mass $\mathcal{M}$. Taking $(\mathcal{M}, \eta)$ to be the post-Newtonian mass parameters the total mass and the reduced mass are given by $M=\mathcal{M} \eta^{-3 / 5}, \mu=\mathcal{M} \eta^{2 / 5}$. Note that in the total chirp time $\tau$ of the signal the 1.5 post-Newtonian contribution appears with a negative sign thus shortening the epoch of coalescence.

With the inclusion of higher order post-Newtonian corrections a chirp template is characterised by a set of four parameters which we shall collectively denote by $\lambda^{\mu}, \mu=1, \ldots, 4$. At the first post-Newtonian approximation instead of working with the parameters $\lambda^{\mu}=$ $\left\{t_{a}, \Phi, \mathcal{M}, \eta\right\}$ we can equivalently employ the set $\left\{t_{a}, \Phi, \tau_{0}, \tau_{1}\right\}$ for the purpose of constructing templates. This, as we shall see later, has some advantageous. However, at post-Newtonian orders beyond the first we do not have a unique set of chirp times to work with.

The parameters $t_{a}$ and $\Phi$ are kinematical that fix the origin of the measurement of time and phase, respectively, while the Newtonian and the post-Newtonian chirp times are dynamical parameters in the sense that they dictate the evolution of the phase and the amplitude of the signal. It may be mentioned at this stage that in most of the literature on this subject authors use the set of parameters $\left\{t_{C}, \Phi_{C}, \mathcal{M}, \eta\right\}$ where $t_{C}$ is the instant of coalescence and $\Phi_{C}$ is the phase of the signal at the instant of coalescence. In terms of the chirp times we have introduced, $t_{C}$ is the sum of the total chirp time and the time of arrival and $\Phi_{C}$ is a combination of the various chirp times and $\Phi$ :

$$
\begin{gathered}
t_{C}=t_{a}+\tau_{0}+\tau_{1}-\tau_{1.5}+\tau_{2} ; \\
\Phi_{C}=\Phi+\frac{16 \pi f_{a}}{5} \tau_{0}+4 \pi f_{a} \tau_{1}-5 \pi f_{a} \tau_{1.5}+8 \pi f_{a} \tau_{2} .
\end{gathered}
$$

In the stationary phase approximation the Fourier transform of the restricted first-postNewtonian chirp waveform for positive frequencies is given by [10, 13, 21,22]

$$
\tilde{h}(f)=\mathcal{N} f^{-7 / 6} \exp \left[i \sum_{\mu=1}^{6} \psi_{\mu}(f) \lambda^{\mu}-i \frac{\pi}{4}\right]
$$

where

$$
\mathcal{N}=A \pi^{2 / 3}\left(\frac{2 \tau_{0}}{3}\right)^{1 / 2} f_{a}^{4 / 3}
$$

is a normalisation constant, $\lambda^{\mu}, \mu=1, \ldots, 6$, represent the various post-Newtonian parameters

$$
\lambda^{\mu}=\left\{t_{a}, \Phi, \tau_{0}, \tau_{1}, \tau_{1.5}, \tau_{2}\right\},
$$

and

$$
\begin{aligned}
& \psi_{1}=2 \pi f \\
& \psi_{2}=-1, \\
& \psi_{3}=2 \pi f-\frac{16 \pi f_{a}}{5}+\frac{6 \pi f_{a}}{5}\left(\frac{f}{f_{a}}\right)^{-5 / 3}, \\
& \psi_{4}=2 \pi f-4 \pi f_{a}+2 \pi f_{a}\left(\frac{f}{f_{a}}\right)^{-1}, \\
& \psi_{5}=-2 \pi f+5 \pi f_{a}-3 \pi f_{a}\left(\frac{f}{f_{a}}\right)^{-2 / 3}, \\
& \psi_{6}=2 \pi f-8 \pi f_{a}+6 \pi f_{a}\left(\frac{f}{f_{a}}\right)^{-1 / 3} .
\end{aligned}
$$


For $f<0$ the Fourier transform is computed using the identity $\tilde{h}(-f)=\tilde{h}^{*}(f)$ obeyed by real functions $h(t)$. In addition to the above mentioned parameters we shall introduce an amplitude parameter $\mathcal{A}$ in section III.

\section{A GEOMETRIC APPROACH TO SIGNAL ANALYSIS}

In this section we apply the techniques of differential geometry to the problem of detecting weak signals embedded in noise. In section IIIA we introduce the concept of the signal manifold and elaborate on the relationship of our approach with that of Amari 19]. Our discussion of the vector space of all detector outputs is modeled after the discussion given in [32]. In section III B we deal with the problem of choosing a set of filters for on-line analysis which would optimise the task of detection of the signal. In section III we deal with the dimensionality of the chirp manifold when we incorporate higher order post-Newtonian corrections. It is found, that due to covariances between the parameters, it is possible to introduce an effective dimension which is less than the dimension of the manifold. This has very important implications for the detection problem.

\section{A. Signal manifold}

The output of a gravitational wave detector such as the LIGO, will comprise of data segments, each of duration $T$ seconds, uniformly sampled with a sampling interval of $\Delta$, giving the number of samples in a single data train to be $N=T / \Delta$. Each data train can be considered as a $N$-tuple $\left(x^{0}, x^{1}, \ldots, x^{N-1}\right) x^{i}$ being the value of the output of the detector at time $i \Delta$. The set of all such $N$ tuples constitutes a $N$-dimensional vector space $\mathcal{V}$ where the addition of two vectors is accomplished by the addition of corresponding time samples. For later convenience we allow each sample to take complex values. A natural basis for this vector space is the time basis $\mathbf{e}_{m}^{i}=\delta_{m}^{i}$ where $m$ and $i$ are the vector and component indices respectively. Another basis which we shall use extensively is the Fourier basis which is related to the time basis by a unitary transformation $\hat{U}$ :

$$
\begin{aligned}
& \tilde{\mathbf{e}}_{m}=\hat{U}^{m n} \mathbf{e}_{n}=\frac{1}{\sqrt{N}} \sum_{n=0}^{N-1} \mathbf{e}_{n} \exp \left[\frac{2 \pi i m n}{N}\right], \\
& \mathbf{e}_{m}=\hat{U}^{\dagger m n} \tilde{\mathbf{e}}_{n}=\frac{1}{\sqrt{N}} \sum_{n=0}^{N-1} \tilde{\mathbf{e}}_{n} \exp \left[-\frac{2 \pi i m n}{N}\right] .
\end{aligned}
$$

All vectors in $\mathcal{V}$ are shown in boldface, and the Fourier basis vectors and components of vectors in the Fourier basis are highlighted with a 'tilde'.

In the continuum case each data train can be expanded in a Fourier series and will contain a finite number of terms in the expansion, as the output will be band limited. The expansion is carried out over the exponential functions $\exp (2 \pi i m t / T)$ which are precisely the Fourier basis vectors defined above. Though the index $m$ takes both positive and negative values corresponding to positive and negative frequencies it is both, possible and convenient to allow $m$ to take only positive values [33]. Thus the vector space $\mathcal{V}$ can be considered as being spanned by the $N$ Fourier basis vectors implying immediately that the number of independent vectors in the time basis to be also $N$. This is the content of the Nyquist theorem which states that it is sufficient to sample the data at a frequency which is twice as large as the bandwidth of a real valued signal, where the bandwidth refers to the range of positive frequencies over which the signal spectrum is non zero. This factor of two does not appear in the vector space picture as we allow in general for complex values for the components in the time basis.

A gravitational wave signal from a coalescing binary system can be characterised by a set of parameters $\boldsymbol{\lambda}=\left(\lambda^{0}, \lambda^{1}, \ldots, \lambda^{p-1}\right)$ belonging to some open set of the $p$-dimensional real space $R^{p}$. The set of such signals $\mathbf{s}(t ; \boldsymbol{\lambda})$ constitutes a $p$-dimensional manifold $\mathcal{S}$ which is embedded in the vector space $\mathcal{V}$. The parameters of the binary act as coordinates on the 
manifold. The basic problem of signal analysis is thus to determine whether the detector output vector $\mathbf{x}$ is the sum of a signal vector and a noise vector, $\mathbf{x}=\mathbf{s}+\mathbf{n}$, or just the noise vector, $\mathbf{x}=\mathbf{n}$, and furthermore to identify which particular signal vector, among all possible. One would also like to estimate the errors in such a measurement.

In the absence of the signal the output will contain only noise drawn from a stochastic process which can be described by a probability distribution on the vector space $\mathcal{V}$. The covariance matrix of the noise $C^{i j}$ is defined as,

$$
C^{i j}=\overline{n^{i} n^{* j}}
$$

where an * denotes complex conjugation and an overbar denotes an average over an ensemble. If the noise is assumed to be stationary and ergodic then there exists a noise correlation function $K(t)$ such that $C_{i j}=K(|i-j| \Delta)$. In the Fourier basis it can be shown that the components of the noise vector are statistically independent [11] and the covariance matrix in the Fourier basis will contain only diagonal terms whose values will be strictly positive: $\tilde{C}_{i i}=\overline{\tilde{n}^{i} \tilde{n}^{* i}}$. This implies that the covariance matrix has strictly positive eigenvalues. The diagonal elements of this matrix $\tilde{C}_{i i}$ constitute the discrete representation of the power spectrum of the noise $S_{n}(f)$.

We now discuss how the concept of matched filtering can be used to induce a metric on the signal manifold. The technique of matched filtering involves correlating the detector output with a bank of filters each of which is tuned to detect the gravitational wave from a binary system with a particular set of parameters. The output of the filter, with an impulse response $\mathbf{q}$, is given in the discrete case as

$$
c_{(m)}=\frac{1}{\sqrt{N}} \sum_{n=0}^{N-1} \tilde{x}^{n} \tilde{q}^{* n} \exp [-2 \pi i m n / N] .
$$

The SNR $(\rho)$ at the output is defined to be the mean of $c_{(m)}$ divided by the square root of its variance:

$$
\rho \equiv \frac{\overline{c_{(m)}}}{\left[\overline{\left(c_{(m)}-\overline{c_{(m)}}\right)^{2}}\right]^{1 / 2}} .
$$

By maximising $\rho$ we can obtain the expression for the matched filter $\mathbf{q}_{(\mathbf{m})}$ matched to a particular signal $\mathbf{s}\left(t ; \lambda^{\mu}\right)$ as

$$
\tilde{q}_{(m)}^{n}\left(\lambda^{\mu}\right)=\frac{\tilde{s}^{n}\left(\lambda^{\mu}\right) \exp [2 \pi i m n / N]}{\tilde{C}_{n n}},
$$

where $\rho$ has been maximised at the $m^{\text {th }}$ data point at the output and where $\mu=1,2, \ldots, p$ where $p$ is the number of parameters of the signal. We now introduce a scalar product in $\mathcal{V}$. For any two vectors $\mathbf{x}$ and $\mathbf{y}$,

$$
\langle\mathbf{x}, \mathbf{s}\rangle=\sum_{i, j=0}^{N-1} C_{i j}^{-1} x^{i} y^{j}=\sum_{n=0}^{N-1} \frac{\tilde{x}^{n} \tilde{s}^{* n}\left(\lambda^{\mu}\right)}{\tilde{C}_{n n}} .
$$

In terms of this scalar product, the output of the matched filter $\mathbf{q}$, matched to a signal $\mathbf{s}\left(\lambda^{\mu}\right)$, can be written as,

$$
c_{(m)}\left(\lambda^{\mu}\right)=\frac{1}{\sqrt{N}}\langle\mathbf{x}, \mathbf{s}\rangle
$$

As $\tilde{C}_{i i}$ is strictly positive the scalar product defined is positive definite. The scalar product defined above on the vector space $\mathcal{V}$ can be used to define a norm on $\mathcal{V}$ which in turn can be used to induce a metric on the manifold. The norm of a vector $\mathbf{x}$ is defined as $\|\mathbf{x}\|=\langle\mathbf{x}, \mathbf{x}\rangle^{1 / 2}$. The ratio for the matched filter can be calculated to give $\rho=\langle\mathbf{s}, \mathbf{s}\rangle^{1 / 2}$. The norm of the noise vector will be a random variable $\langle\mathbf{n}, \mathbf{n}\rangle^{1 / 2}$ with a mean of $\sqrt{N}$ as can be 
seen by writing the expression for the norm of the noise vector and subsequently taking an ensemble average.

The distance between two points infinitesimally separated on $\mathcal{S}$ can be expressed as a quadratic form in the differences in the values of the parameters at the two points:

$$
\begin{aligned}
g_{\mu \nu} d \lambda^{\mu} d \lambda^{\nu} & \equiv\left\|\mathbf{s}\left(\lambda^{\mu}+d \lambda^{\mu}\right)-\mathbf{s}\left(\lambda^{\mu}\right)\right\|=\left\|\frac{\partial \mathbf{s}}{\partial \lambda^{\mu}} d \lambda^{\mu}\right\| \\
& =\left\langle\frac{\partial \mathbf{s}}{\partial \lambda^{\mu}}, \frac{\partial \mathbf{s}}{\partial \lambda^{\nu}}\right\rangle d \lambda^{\mu} d \lambda^{\nu}
\end{aligned}
$$

The components of the metric in the coordinate basis are seen to be the scalar products of the coordinate basis vectors of the manifold.

Since the number of correlations we can perform on-line is finite, we cannot have a filter corresponding to every signal. A single filter though matched to a particular signal will also detect signals in a small neighbourhood of that signal but with a loss in the SNR. The metric on the manifold quantifies the drop in the correlation in a neighbourhood of the signal chosen. Taking the output vector to be $\mathbf{x}$ and two signal vectors $\mathbf{s}(\boldsymbol{\lambda})$ and $\mathbf{s}(\boldsymbol{\lambda}+\mathbf{d} \boldsymbol{\lambda})$ and using Schwarz's inequality we have,

$$
\begin{aligned}
\langle\mathbf{x}, \mathbf{s}(\boldsymbol{\lambda}+\mathbf{d} \boldsymbol{\lambda})\rangle-\langle\mathbf{x}, \mathbf{s}(\boldsymbol{\lambda})\rangle=\langle\mathbf{x}, \mathbf{s}(\boldsymbol{\lambda}+d \boldsymbol{\lambda})-\mathbf{s}(\boldsymbol{\lambda})\rangle & \leq\|\mathbf{x}\|\|\mathbf{s}(\boldsymbol{\lambda}+d \boldsymbol{\lambda})-\mathbf{s}(\boldsymbol{\lambda})\| \\
& =\|\mathbf{x}\| g_{\mu \nu} d \lambda^{\mu} d \lambda^{\nu}
\end{aligned}
$$

As is apparent the drop in the correlation can be related to the metric distance on the manifold between the two signal vectors.

We now discuss Amari's 19] work in the context of using differential geometry in statistics and elaborate on the relationship with the approach we have taken. The set of parametrised probability distributions corresponding to a statistical model constitute a manifold. The parameterized probability distributions in the context of signal analysis of gravitational waves from coalescing binaries are the ones which specify the probability that the output vector will lie in a certain region of the vector space $\mathcal{V}$ given that a signal $\mathbf{s}(t ; \boldsymbol{\lambda})$ exists in the output which we denote as $p(\mathbf{x} \mid \mathbf{s}(t ; \boldsymbol{\lambda}))$ Since it is not our intention to develop Amari's approach any further we will be brief and will make all the mathematical assumptions such as infinite differentiability of functions, interchangeability of the differentiation and expectation value operators, etc.

The set of probability distributions $p(\mathbf{x} \mid \mathbf{s}(\boldsymbol{\lambda}))$ where $\boldsymbol{\lambda} \in R^{p}$, constitutes a manifold $\mathcal{P}$ of dimension $p$. At every point on this manifold we can construct a tangent space $T^{0}$ on which we can define the coordinate basis vectors as $\partial_{\mu}=\frac{\partial}{\partial \lambda^{\mu}}$. Any vector $\mathbf{A}$ in this tangent space can be written as a linear combination of these coordinate basis vectors. We now define $p$ random variables $\sigma_{\mu}=-\frac{\partial}{\partial \lambda^{\mu}} \log (p(\mathbf{x} \mid \mathbf{s}(\boldsymbol{\lambda})))$. It can easily be shown that $\overline{\sigma_{\mu}}=0$. We assume that these $p$ random variables are linearly independent. By taking all possible linear combinations of these random variables we can construct another linear space $T^{1}$. Each vector $\mathbf{B}$ in $T^{1}$ can be written as $\mathbf{B}=B^{\mu} \sigma_{\mu}$. The two vector spaces $T^{0}$ and $T^{1}$ are isomorphic to each other, which can be shown explicitly by making the correspondence $\sigma_{\mu} \leftrightarrow \partial_{\mu}$. The vector space $T^{1}$ has a natural inner product defined on it which is the covariance matrix of the $p$ random variables $\sigma_{\mu}$. This scalar product can be carried over to $T^{0}$ using the correspondence stated above. The metric on the manifold can be defined by taking the scalar product of the coordinate basis vectors

$$
g_{\mu \nu}=\left\langle\partial_{\mu}, \partial_{\nu}\right\rangle=\overline{\sigma_{\mu} \sigma_{\nu}}
$$

In statistical theory the above matrix $g_{\mu \nu}$ is called the Fisher information matrix. We will also denote the Fisher matrix, as is conventional, as $\Gamma_{\mu \nu}$. It is clearly seen that orthogonality between vectors in the tangent space of the manifold is related to statistical independence of random variables in $T^{1}$.

If we take the case of Gaussian noise the metric defined above is identical to the one obtained on the signal manifold by matched filtering. Gaussian noise can be described by the distribution, 


$$
p(\mathbf{n})=\frac{\exp \left[-\frac{1}{2} \sum_{j, k=0}^{N-1} C_{j k}^{-1} n^{j} n^{k *}\right]}{\left[(2 \pi)^{N} \operatorname{det}\left[C_{i j}\right]\right]^{1 / 2}}=\frac{\exp \left[-\frac{1}{2} \sum_{j, k=0}^{N-1} \tilde{C}_{j k}^{-1} \tilde{n}^{j} \tilde{n}^{k *}\right]}{\left[(2 \pi)^{N} \operatorname{det}\left[\tilde{C}_{i j}\right]\right]^{1 / 2}}=\frac{\exp \left[-\frac{1}{2} \sum_{i=0}^{N-1} \frac{\tilde{n}^{i} \tilde{n}^{i *}}{\tilde{C}_{i i}}\right]}{\left[(2 \pi)^{N} \operatorname{det}\left[\tilde{C}_{i j}\right]\right]^{1 / 2}},
$$

where in the last step we have used the diagonal property of the matrix $\tilde{C}^{i j}$ which implies that $\tilde{C}_{i i}^{-1}=1 / \tilde{C}_{i i}$.

As the noise is additive $p(\mathbf{x} \mid \mathbf{s}(\boldsymbol{\lambda}))$ can be written as $p(\mathbf{x}-\mathbf{s}(\boldsymbol{\lambda}))$. Assuming Gaussian noise we can write the expressions for the random variables $\sigma_{i}$ as,

$$
\sigma_{\mu}=\left(\frac{1}{2}\right) \frac{\partial}{\partial \lambda^{\mu}}\langle\mathbf{x}-\mathbf{s}(\boldsymbol{\lambda}), \mathbf{x}-\mathbf{s}(\boldsymbol{\lambda})\rangle=\left\langle\mathbf{n}, \frac{\partial}{\partial \lambda^{\mu}} \mathbf{s}(\boldsymbol{\lambda})\right\rangle,
$$

where in the last step we have used $\mathbf{x}=\mathbf{s}(\boldsymbol{\lambda})+\mathbf{n}$. The covariance matrix for the random variables $\sigma_{\mu}$ can be calculated to give

$$
\overline{\sigma_{\mu} \sigma_{\nu}}=\left\langle\frac{\partial}{\partial \lambda^{\mu}} \mathbf{s}(\boldsymbol{\lambda}), \frac{\partial}{\partial \lambda^{\nu}} \mathbf{s}(\boldsymbol{\lambda})\right\rangle,
$$

which is the same metric as defined over the signal manifold. Thus, both the manifolds $\mathcal{S}$ and $\mathcal{P}$ are identical with respect to their metrical properties. We will henceforth restrict our attention to the signal manifold $\mathcal{S}$.

For the purpose of our analysis we will choose a minimal set of parameters characterizing the gravitational wave signal from a coalescing binary. We consider only the first post-Newtonian corrections. In section II we have already introduced the four parameters $\lambda^{\mu}=\left\{t_{a}, \Phi, \tau_{0}, \tau_{1}\right\}$. We now introduce an additional parameter for the amplitude and call it $\lambda^{0}=\mathcal{A}$. The signal can now be written as $\tilde{s}(f ; \boldsymbol{\lambda})=\mathcal{A} h\left(f ; t_{a}, \Phi, \tau_{0}, \tau_{1}\right)$, where, $\boldsymbol{\lambda} \equiv\left\{\mathcal{A}, t_{a}, \Phi, \tau_{0}, \tau_{1}\right\}$. Numerically the value of the parameter $\mathcal{A}$ will be the same as that of the SNR obtained for the matched filter. We can decompose the signal manifold into a manifold containing normalised chirp waveforms and a one-dimensional manifold corresponding to the parameter $\mathcal{A}$. The normalised chirp manifold can therefore be parameterized by $\left\{t_{a}, \Phi, \tau_{0}, \tau_{1}\right\}$. This parameterization is useful as the coordinate basis vector $\frac{\partial}{\partial \mathcal{A}}$ will be orthogonal to all the other basis vectors as will be seen below.

In order to compute the metric, and equivalently the Fisher information matrix, we use the continuum version of the scalar product as given in [20], except that we use the two sided power spectral density. This has the advantage of showing clearly the range of integration in the frequency space though we get the same result using the discrete version of the scalar product. Using the definition of the scalar product we get

$$
g_{\mu \nu}=\int_{f_{a}}^{\infty} \frac{d f}{S_{n}(f)} \frac{\partial \tilde{s}(f ; \boldsymbol{\lambda})}{\partial \lambda^{\mu}} \frac{\partial \tilde{s}^{*}(f ; \boldsymbol{\lambda})}{\partial \lambda^{\nu}}+\text { c.c. }
$$

Recall that in the stationary phase approximation the Fourier transform of the coalescing binary waveform is given by $\tilde{h}(f)=\mathcal{N} f^{-7 / 6} \exp \left[i \sum_{\mu} \psi_{\mu}(f) \lambda^{\mu}\right]$ and $\tilde{s}(f)=\mathcal{A} \tilde{h}(f)$, where $\psi_{\mu}(f)$ are given by equations (2.18-2.23) $, \mu=1, \ldots, 4$ and $\lambda^{\mu}=\left\{t_{a}, \Phi, \tau_{0}, \tau_{1}\right\}$. Note, in particular, that in the phase of the waveform the parameters occur linearly thus enabling a very concise expression for the components of $g_{\mu \nu}$. The various partial derivatives are given by

$$
\frac{\partial \tilde{s}(f ; \boldsymbol{\lambda})}{\partial \lambda^{\mu}}=i \psi_{\mu}(f) \tilde{s}(f ; \boldsymbol{\lambda}),
$$

where we have introduced $\psi_{0}=-i / \mathcal{A}$. On substituting the above expressions for the partial derivatives in eq. (3.17) we get,

$$
g_{\mu \nu}=\left\langle\psi_{\mu} h, \psi_{\nu} h\right\rangle=2 \int_{f_{a}}^{\infty} \frac{\psi_{\mu}(f) \psi_{\nu}^{*}(f)|\tilde{h}(f)|^{2}}{S_{n}(f)} d f
$$


The above definition of the amplitude parameter $\mathcal{A}$, as in Culter and Flannagan [22], disjoins the amplitude of the waveform from the rest of the parameters. Since $\psi_{0}$ is pure imaginary and $\psi_{\mu}$ 's are real, it is straightforward to see from eq. (3.19) that

$$
g_{00}=1 \text { and } g_{0 \mu}=0 ; \quad \mu=1, \ldots, 4 .
$$

The rest of the components $g_{\mu \nu}$ are seen to be independent of all the parameters except $\mathcal{A}$ i.e. $g_{\mu \nu} \propto \mathcal{A}^{2}$. As $\mathcal{A}$ is unity for the normalised manifold the metric on the normalised manifold is flat. This implies not only that the manifold is intrinsically flat (in the stationary phase approximation) but also that the coordinate system used is Cartesian. If instead of the chirp time $\tau_{0}$ we use the parameter $\mathcal{M}$ then the metric coefficients will involve that parameter and the coordinate system will no longer remain Cartesian.

\section{B. Choice of filters}

We now use the formalism developed to tackle the issue of optimal filter placement. Till now, it has been thought necessary to use a finite subset of the set of chirp signals as templates for detection. We show that this is unduly restrictive. We suggest a procedure by which the detection process can be made more 'efficient' by moving the filters out of the manifold. It must be emphasised that the algorithm presented below is both, simplistic and quite adhoc and is not necessarily the best. Moreover, we have implemented the algorithm only for the Newtonian case where the computational requirements are not very heavy. The signal manifold corresponding to higher post-Newtonian corrections will be a higher dimensional manifold and here the computational requirements will be substantial. The choice of optimal filters which span the manifold will then be crucial.

Detection of the coalescing binary signal involves computing the scalar product of the output of the detector with the signal vectors. Subsequently one would have to maximise the correlations over the parameters and the number so obtained would serve as the statistic on the basis of which we can decide whether a signal is present in the given data train. Geometrically, this maximization corresponds to minimizing the angle between the output vector and the vectors corresponding to the normalised signal manifold. Using the cosine formula,

$$
\cos (\theta)=\frac{\langle\mathbf{s}(\boldsymbol{\lambda}), \mathbf{x}\rangle}{\|\mathbf{x}\|\|\mathbf{s}(\boldsymbol{\lambda})\|}=\frac{\|\mathbf{s}(\boldsymbol{\lambda})\|^{2}+\|\mathbf{x}\|^{2}-\|\mathbf{x}-\mathbf{s}(\boldsymbol{\lambda})\|^{2}}{2\|\mathbf{x}\|\|\mathbf{s}(\boldsymbol{\lambda})\|},
$$

it is clear that as $\|\mathbf{s}(\boldsymbol{\lambda})\|$ is unity for the vectors belonging to the normalised signal manifold, maximising the scalar product is equivalent to minimising $\|\mathbf{x}-\mathbf{s}(\boldsymbol{\lambda})\|$ which is the distance between the tip of the output vector and the manifold.

Given the constraints of computational power one would be able to evaluate only a finite number of these scalar products, say $n_{F}$, in a certain amount of time depending on the data train length and the padding factor. It is therefore necessary to be able to choose the $n_{F}$ filters in such a manner that the detection probability is maximal. We will need efficient on-line data analysis for two reasons: (i) To isolate those data trains which have a high probability of containing a signal and (ii) to determine the parameters of the binary early on during the inspiral and to use them for dynamical recycling techniques.

Due to the finiteness of the filter spacing the signal parameters will in general not correspond to any of the $n_{F}$ filters chosen and this will lead to a drop in the maximum possible correlation. Till now attention has been focussed on identifying a set of optimal set of filters which are a discrete subset of the manifold. If detection is the sole purpose then the differential geometric picture suggests that confining the filter vectors to the signal manifold is an unnecessary restriction and in fact non optimal. Thus it is worthwhile to explore making a choice of filters outside the manifold. The filter vectors will thus belong to $\mathcal{V}$ but will not, in general, correspond to any signal. It is, of course, true that we are sacrificing on the maximum possible correlation obtainable (when the signal's parameters coincide with those of the filter). Thus the problem essentially is to select $n_{F}$ filter vectors which optimize the detection the efficiency of which depends upon the properties of the manifold. 
In general a single filter vector would have to pick up signals over a region of the manifold. The extent of this region is determined by fixing a threshold on the correlation between the filter and any signal in the region. We will denote this threshold by $\eta$, where $\eta$ takes a value which is close to, but less than unity. The typical value suggested for $\eta$ is $\sim 0.8$, [13]. For a given filter $\mathbf{q}$ and a threshold $\eta$ the region on the manifold corresponding to the filter will be denoted as $\mathcal{S}_{\mathbf{q}}(\eta)$, where $\mathcal{S}_{\mathbf{q}}(\eta) \subset \mathcal{S}$. Geometrically this region is the intersection of an open ball in $\mathcal{V}$ of radius $2^{1 / 2}(1-\eta)^{1 / 2}$ (using the distance defined by the scalar product), with center $\mathbf{q}$, and the manifold. The $n_{F}$ filters taken together would have to 'span' the manifold which means that the union of the regions covered by each filter would be the manifold itself i.e., $\bigcup_{\mathbf{q}} \mathcal{S}_{\mathbf{q}}(\eta)=\mathcal{S}$. If the filter $\mathbf{q}$ lies on the manifold, then the correlation function $c_{\mathbf{q}}(\boldsymbol{\lambda})=\langle\mathbf{s}(\boldsymbol{\lambda}), \mathbf{q}\rangle$ will reach its maximum value of unity in $\mathcal{S}_{\mathbf{q}}(\eta)$ when $\mathbf{q}=\mathbf{s}(\boldsymbol{\lambda})$ and will fall off in all directions. This means that the signals in the region which are further away from the filter are less likely to be picked up as compared to those in the immediate neighbourhood of the filter $\mathbf{q}$.

We assume that a finite subset of the normalised signal manifold has been chosen to act as filters by some suitable algorithm 13], which taken together span the manifold. The number of filters will be determined by the available computing power. Consider one of these filters $\mathbf{q}$, the region corresponding to it for a threshold of $\eta, \mathcal{S}_{\mathbf{q}}(\eta)$, and an arbitrary normalised vector $\mathbf{q}_{o}$ which belongs to $\mathcal{V}$ but not necessarily $\mathcal{S}$. By correlating the vector $\mathbf{q}_{o}$ with vectors in $\mathcal{S}_{\mathbf{q}}(\eta)$ we obtain the correlation function $c_{\mathbf{q}_{o}}(\boldsymbol{\lambda})=\left\langle\mathbf{s}(\boldsymbol{\lambda}), \mathbf{q}_{o}\right\rangle$. We select that $\mathbf{q}_{o}$ to serve as a more optimal filter which maximises the average of this correlation function:

$$
\left\langle\mathbf{s}(\boldsymbol{\lambda}), \mathbf{q}_{o}\right\rangle_{a v}=\frac{1}{\int_{\mathcal{S}_{\mathbf{q}}(\eta)} \sqrt{g} d^{p} \lambda} \int_{\mathcal{S}_{\mathbf{q}}(\eta)}\left\langle\mathbf{s}(\boldsymbol{\lambda}), \mathbf{q}_{o}\right\rangle \sqrt{g} d^{p} \lambda=\left\langle\int_{\mathcal{S}_{\mathbf{q}}(\eta)} \mathbf{s}(\boldsymbol{\lambda}) d^{p} \lambda, \mathbf{q}_{o}\right\rangle,
$$

where $g=\operatorname{det}\left[g_{\mu \nu}\right]$. In the last step above the integration and the scalar product operations in the above equation have been interchanged. Moreover, for the normalised chirp manifold the metric does not depend upon the parameters in the coordinate system we have chosen and therefore, $g_{\mu \nu}$ is a constant and the factor $\sqrt{g}$ cancels. We now use Schwarz's inequality to maximise the average correlation to obtain,

$$
\mathbf{q}_{o}=\mathcal{N} \int_{\mathcal{S}_{\mathbf{q}}(\eta)} \mathbf{s}(\boldsymbol{\lambda}) d^{p} \lambda
$$

where $\mathcal{N}$ is just a normalisation constant.

We implemented the above algorithm for filter placement for the case of Newtonian signals with certain modifications. The normalised chirp waveform consists of three parameters $\left(\Phi, t_{a}, \tau_{n}\right)$. If we keep $t_{a}$ and $\tau_{n}$ fixed then the tip of the signal vector traces out a circle as we vary $\Phi$. As any circle lies on a plane we can express a signal vector as a linear sum of two vectors where the two vectors differ only in the phase parameter and we take this phase difference to be $\pi / 2$. Thus we need only two mutually orthogonal filters to span the phase parameter. The time of arrival parameter $t_{a}$ is also a 'convenient parameter' as by the use of fast Fourier transforms the correlations for arbitrary time of arrivals can be performed at one go. It is therefore not profitable for us to maximise the average correlation over the phase parameter $\Phi$ and the time of arrival $t_{a}$.

In view of the above restrictions we modified the the filter placement algorithm. We consider the correlation function for the case when the filter vector is on the manifold. We define the 'line of curvature' to be the curve on the manifold along which the correlation function falls the least. Figure (1) illustrates the correlation function plotted as a function of $\tau_{0}$ along the line of curvature. It is seen from the contour diagram of the numerically computed correlation function that the line of curvature lies nearly on the submanifold $t_{a}+\tau_{n}=$ constant, of the normalised chirp manifold. We take two curves passing through the point $\mathbf{q}$ in the region $\mathcal{S}_{\mathbf{q}}(\eta)$ :

$$
\begin{aligned}
& \text { 1. } t_{a}+\tau_{n}=\text { constant, } \Phi=0 \text { and } \\
& \text { 2. } t_{a}+\tau_{n}=\text { constant, } \Phi=\pi / 2 .
\end{aligned}
$$


We obtain one filter for each of the two curves by evaluating eq. 3.23 where the domains of integration correspond to the segments of the curves defined.

Having determined the two filters we again plot the correlation function along the line of curvature as a function of $\tau_{0}$ in figure (11). The region of the manifold selected corresponds to a range of 5.8 secs to 6.0 secs in the parameter $\tau_{0}$. It is interesting to note that we could have translated these values keeping the difference same without affecting our results. It can be seen that the correlation has a minimum at the center. In order to get a flatter correlation curve we select a linear combination of the original filter and the one obtained by averaging with suitable weights attached to each filter. This performs reasonably well as shown by the thick curve in the figure. The importance of having a flatter correlation function lies in the fact all the signals in a region can be picked up with equal efficiency and the drop in the maximum possible correlation can be compensated for by lowering the threshold. The average correlation obtained for the optimal filter is only marginally better than that obtained for the filter placed on the manifold.

In the discussion above, we had started with a fixed number of filters $n_{F}$ on the manifold and obtained another set of $n_{F}$ filters which performs marginally better than the former set. Equivalently we can try to increase the span of each filter retaining the same threshold but reducing the number of filters required. In Figure 1 we observe that the optimal filter chosen spans the entire region considered with a threshold greater than 0.9 , whereas the filter on the manifold spans about half the region at the same threshold. This indicates that by moving the filters out of the manifold in the above manner it may be possible to reduce the number of filters by a factor of two or so. One must however, bear in mind that the bank of filters obtained in this way are not optimal. There is scope to improve the scheme further. Our analysis is indicative of this feature.

\section{Effective dimensionality of the parameter space of a second order post-Newtonian waveform}

It has already been shown that the first post-Newtonian waveform is essentially onedimensional [28]. We argue in this subsection that even the second post-Newtonian waveform is essentially one-dimensional and a one-dimensional lattice suffices to filter the waveform.

A Newtonian waveform is characterised by a set of three parameters consisting of the time of arrival, the phase of the signal at the time of arrival and chirp mass (equivalently, Newtonian chirp time). In this case, for the purpose of detection, one essentially needs to employ a one-dimensional lattice of filters corresponding to the chirp mass, the time of arrival being taken care by the fast Fourier transform algorithm and the phase being determined using a two-dimensional basis of orthogonal templates. When post-Newtonian corrections are included in the phase of the waveform the number of parameters increases apparently implying that one needs to use a two-dimensional lattice of filters corresponding to, say, the chirp and reduced masses (equivalently the Newtonian and post-Newtonian chirp times) which in turn means that the number of templates through which the detector output needs to be filtered goes up by several orders of magnitude. One of us (BSS) has recently shown that for the purpose of detection it is sufficient to use a one-dimensional lattice of filters even after first post-Newtonian corrections are included in the phase of the waveform and the relevant parameter here is the sum of the Newtonian and post-Newtonian chirp times. What happens when corrections beyond the first post-Newtonian order are incorporated in the phase of the waveform?

The coalescing binary waveform is now available up to second post-Newtonian order [31,34. Blanchet et al. argue that the phase correction due to the second order postNewtonian $(2 \mathrm{PN})$ term induces an accumulated difference of 10 cycles in a total of 16000 . Consequently, it is important to incorporate the $2 \mathrm{PN}$ terms in the templates. When the $2 \mathrm{PN}$ terms are included it is useful to consider that the full waveform is parameterised by three additional parameters, corresponding to the chirp times at the 1, 1.5 and $2 \mathrm{PN}$ order (cf. Sec II), as compared to the Newtonian waveform. Of course, as far as the detection problem is concerned there is only one additional parameter since the chirp times are all functions of the two masses of the binary. However, for the purpose of testing general 
relativity one can consider each of the chirp times to be independent of the rest [5.23. Our problem now is to find the dimensionality of the parameter space of a $2 \mathrm{PN}$ waveform. To this end we consider the ambiguity function $C\left(\boldsymbol{\lambda}^{\prime}, \boldsymbol{\lambda}\right)$ which is nothing but the correlation function of two normalised waveforms one of whose parameters $(\boldsymbol{\lambda})$ are varied by holding the parameters of the other fixed $\left(\boldsymbol{\lambda}^{\prime}\right)$ :

$$
C\left(\boldsymbol{\lambda}^{\prime}, \boldsymbol{\lambda}\right)=\left\langle q\left(\boldsymbol{\lambda}^{\prime}\right), q(\boldsymbol{\lambda})\right\rangle ;\left\langle q\left(\boldsymbol{\lambda}^{\prime}\right), q\left(\boldsymbol{\lambda}^{\prime}\right)\right\rangle=\langle q(\boldsymbol{\lambda}),(\boldsymbol{\lambda})\rangle=1 .
$$

It is useful to think of $\boldsymbol{\lambda}^{\prime}$ to be the parameters of a template and $\boldsymbol{\lambda}$ to be that of a signal. With this interpretation the ambiguity function simply gives the span of a filter in the parameter space.

The ambiguity function for the full waveform is a four-dimensional surface since there are four independent parameters. To explore the effective dimensionality of the parameter we consider the set of parameters to be $\left\{t_{a}, \Phi, m_{1}, m_{2}\right\}$, where $m_{1}$ and $m_{2}$ are the two masses of the binary. We have shown the contours of the ambiguity function maximised over $t_{a}$ and $\Phi$ (since these two parameters do not explicitly need a lattice of templates) in Fig. 2. The template at the centre of the plot corresponds to a binary waveform with $m_{1}=m_{2}=1.4 M_{\odot}$ and the signal parameters are varied over the entire astrophysically interesting range of masses: $m_{1}, m_{2} \in[1.4,10] M_{\odot}$. From these figures we find that the ambiguity function is almost a constant along a particular line in the $m_{1}-m_{2}$ plane. This means that a template at the centre of the grid spans a relatively large area of the parameter space by obtaining a correlation very close to unity for all signals whose masses lie on the curve along which the ambiguity function roughly remains a constant. It turns out that the equation of this curve is given by

$$
\tau_{0}+\tau_{1}-\tau_{1.5}+\tau_{2}=\text { const. }
$$

Let us suppose we begin with a two-dimensional lattice of filters corresponding to a certain grid (albeit, nonuniform) laid in the $m_{1}-m_{2}$ plane. Several templates of this set will have their total chirp time the same. Now with the aid of just one template out of all those that have the same chirp time we can effectively span the region that is collectively spanned by all such filters. More precisely, we will not have an appreciable loss in the SNR in replacing all templates of a given total chirp time by one of them. Consequently, the signal manifold can be spanned by a one-dimensional lattice of templates.

\section{ESTIMATION OF PARAMETERS}

In this section we discuss the accuracy at which the various parameters of a coalescing binary system of stars can be estimated. All our results are for a single interferometer of the initial LIGO-type which has a lower frequency cutoff at $40 \mathrm{~Hz}$. At present it is beyond the computer resources available to us to carry out a simulation for the advanced LIGO. In the first part of this section we briefly review the well known results obtained for the variances and covariances in the estimation of parameters using analytical methods. Analytical methods assume that the SNR is sufficiently large (the so-called strong signal approximation) and implicitly use a continuum of the parameter space. In reality, however, these assumptions are not necessarily valid and hence it is essential to substantiate the results obtained using analytical means by performing numerical simulations. In the second part of this section we present an exhaustive discussion of the Monte Carlo simulations we have performed to compute the errors and covariances of different parameters. As we shall discuss below the computation of errors using the covariance matrix is erroneous even at an SNR of 10-20. Our estimation of 1- $\sigma$ uncertainty in the various parameters, at low SNRs, is substantially larger than those computed using the covariance matrix. However, for high values of the SNR (>25-30) Monte Carlo estimation agrees with the analytical results. 


\section{A. Covariance matrix}

In recent years a number of authors have addressed issues related to the variances expected in the parameter estimation [5, 18,20 27], In the standard method of computing the variances in the estimation of parameters one makes the assumption that the SNR is so large that with the aid of such an approximation one can first construct the Fisher information matrix $\Gamma_{\mu \nu}$ and then take its inverse to obtain the covariance matrix $C_{\mu \nu}$. In the strong signal approximation the Fisher information matrix and the covariance matrix are given by

$$
g_{\mu \nu}=\Gamma_{\mu \nu}=\left\langle\frac{\partial \mathbf{s}}{\partial \lambda^{\mu}}, \frac{\partial \mathbf{s}}{\partial \lambda^{\nu}}\right\rangle ; \quad C_{\mu \nu}=\Gamma^{-1} \mu \nu .
$$

As we have seen before the Fisher information and consequently the covariance matrix is block diagonal and hence there is no cross-talk, implying vanishing of the covariances between the amplitude and the other parameters. Consequently, we need not construct, for the purpose of Weiner filtering, templates corresponding to different amplitudes.

The judicious choice of parameters has also allowed a very elegant expression for the Fisher information matrix. It is particularly interesting to note that the information matrix does not depend on the values of the various parameters, except for the amplitude parameter, and hence is a constant as far as the parameter space of the normalised waveforms is considered.

For the purpose of numerical simulations it is convenient to choose the set $\lambda^{\mu}=$ $\left\{\mathcal{A}, t_{a}, \Phi, \tau_{0}, \tau_{1}\right\}$ where $\mathcal{A}$ is the amplitude parameter, $t_{a}$ and $\Phi$ are the time of arrival of the signal and its phase at the time of arrival, and $\tau_{0}$ and $\tau_{1}$ are the Newtonian and the post-Newtonian coalescence times. For noise in realistic detectors, such as LIGO, the elements of the Fisher information matrix cannot be expressed in a closed form and, for the set of parameters employed, it is not useful to explicitly write down the covariance matrix in terms of the various integrals since the errors and covariances do not have any dependence on the parameters. We thus evaluate the information matrix numerically and then take its inverse to obtain the covariance matrix. Instead of dealing with the covariance matrix $C$ it more instructive to work with the matrix of standard deviations and correlation coefficients $D$ which is related to the former by

$$
D_{\mu \nu}= \begin{cases}\sqrt{C_{\mu \nu}}, & \text { if } \mu=\nu \\ C_{\mu \nu} /\left(\sigma_{\mu} \sigma_{\nu}\right) & \text { if } \mu \neq \nu\end{cases}
$$

where $\sigma_{\mu}=D_{\mu \mu}$ is the 1- $\sigma$ uncertainty in the parameter $\lambda_{\mu}$. The off-diagonal elements of $D$ take on values in the range $[-1,1]$ indicating how two different parameters are correlated: For $\mu \neq \nu, D_{\mu \nu}=1$, indicates that the two are perfectly correlated, $D_{\mu \nu}=-1$ means that they are perfectly anticorrelated and $D_{\mu \nu}=0$ implies that they are uncorrelated. Since the information matrix is block-diagonal, the amplitude parameter is totally uncorrelated with the rest and thus an error in the measurement of $\mathcal{A}$ will not reflect itself as an error in the estimation of the other parameters and vice versa. In contrast, as we shall see below, Newtonian chirp time is strongly anticorrelated to post-Newtonian chirp time, which implies that if in a given experiment $\tau_{0}$ happens to be estimated larger than its true value then it is more likely that $\tau_{1}$ will be estimated to be lower than its actual value. Such correlations are useful as far as detection is concerned since they tend to reduce the number of templates needed in filtering a given signal. On the other hand, strong correlations increase the volume in the parameter space to which an event can be associated at a given confidence level. It seems to be in general true that a given set of parameters do not satisfy the twin properties of having small covariances and reducing the effective dimension of the manifold for the purpose of filtering. We elaborate on this point below.

Given a region in a parameter space, it is useful to know the proper volume (as defined by the metric) of the manifold corresponding to the said region. In choosing a discrete set of filters for the detection problem one has to decide upon the maximum allowable drop in the correlation due to the finite spacing. Once this is fixed, the number of filters can be determined from the total volume of the manifold. For the detection problem it is beneficial to have a small volume whereas if the waveform is parameterized in such a way such that the manifold corresponding to it covers a large volume, then one can determine the parameters 
to a greater accuracy. As a simple example let us consider a two-dimensional toy model: $\boldsymbol{\lambda}=\left\{\lambda_{1}, \lambda_{2}\right\}$. We compare different signal manifolds each corresponding to a different parameterizations of the waveform. We assume that the covariance matrix and its inverse, the Fisher information matrix, to be:

$$
C_{\mu \nu}=\left(\begin{array}{ll}
\sigma_{11} & \sigma_{12} \\
\sigma_{12} & \sigma_{22}
\end{array}\right) \quad \text { and } \quad \Gamma_{\mu \nu}=\left(\begin{array}{ll}
\gamma_{11} & \gamma_{12} \\
\gamma_{12} & \gamma_{22}
\end{array}\right)=\frac{1}{\left(\sigma_{11} \sigma_{22}-\sigma_{12}^{2}\right)}\left(\begin{array}{cc}
\sigma_{22} & -\sigma_{12} \\
-\sigma_{12} & \sigma_{11}
\end{array}\right)
$$

The volume of the manifold corresponding to a region $\mathcal{K}$ of the parameter space is given as,

$$
V_{\mathcal{K}}=\int_{\mathcal{K}} \gamma_{11} \gamma_{22}\left[1-\frac{\gamma_{12}^{2}}{\gamma_{11} \gamma_{22}}\right] d \lambda_{1} d \lambda_{2}=\int_{\mathcal{K}} \gamma_{11} \gamma_{22}[1-\epsilon] d \lambda_{1} d \lambda_{2}
$$

where $\epsilon=\gamma_{12}^{2} /\left(\gamma_{11} \gamma_{22}\right)$ is the correlation coefficient. It can be clearly seen that if the correlation coefficient is small, keeping the variances in the parameters, fixed then the volume of the manifold is maximal. Since the parameters $\tau_{0}$ and $\tau_{1}$ are highly anticorrelated the proper volume corresponding to the region reduces to zero showing that the effective dimensionality of the manifold is less.

Though, in principle, the variances and covariances are independent of the chirp time, in reality there arises an indirect dependence since one terminates a template at a frequency $f=1 /\left(6^{3 / 2} \pi M\right)$ (where $M$ is the total mass of the binary) corresponding to the plunge radius at $a=6 M$. Therefore, larger mass binaries are tracked over a smaller bandwidth so much so that there is less frequency band to distinguish between two chirps of large, but different, total mass. Consequently, at a given SNR the error in the estimation of chirp times is larger for greater mass binaries. This is reflected by the fact that the integrals in eq. (3.17) are somewhat sensitive to the value of the upper cutoff. (This also explains why the errors in the estimation of the chirp and reduced masses are larger for greater mass binaries [21,22].) In the following we assume that the noise power spectral density is that corresponding to the initial LIGO for which a fit has been provided by Finn and Chernoff [21]. For an SNR of 10 the matrix $D$ is given by

$$
D_{\mu \nu}=\left(\begin{array}{rrrr}
1.0 & 0 & 0 & 0 \\
& 8.37 & 0.999 & -0.999 \\
& & 3.16 & -0.998 \\
& & & 8.4
\end{array}\right) \text {, }
$$

for the Newtonian signal, and by

$$
D_{\mu \nu}=\left(\begin{array}{rrrrr}
1.0 & 0 & 0 & 0 & 0 \\
& 20.4 & 0.997 & -0.972 & 0.911 \\
& & 6.7 & -0.954 & 0.881 \\
& & & 45.1 & -0.982 \\
& & & & 25.98
\end{array}\right),
$$

for the first post-Newtonian corrected signal. While computing varianaces and covariances the integrals in equation (3.19) are evaluated by chosing a finite upper limit of $1 \mathrm{kHz}$. In the above matrices the entries are arranged in the order $\left\{\mathcal{A}, t_{a}, \Phi, \tau_{0}\right\}$ in the Newtonian case, $\left\{\mathcal{A}, t_{a}, \Phi, \tau_{0}, \tau_{1}\right\}$ in the post-Newtonian case, off diagonal elements are dimensionless correlation coefficients and, where appropriate, diagonal elements are in ms. The values quoted in the case of the Newtonian waveform are consistent with those obtained using a different set of parameters by Finn and Chernoff [21. In Fig. 3 we have plotted $\sigma$ 's, at an SNR of 10, as a function of the upper frequency cutoff $f_{c}$ for Newtonian and post-Newtonian chirp times and the instant of coalescence $t_{C}$. We see that $\sigma$ is larger for higher mass binaries but this is because we have fixed the SNR. However, if we consider binaries of different total masses, all located at the same distance, then a more massive binary produces a stronger SNR so that in reality it may be possible to determine its parameters more accurately than that of a lighter binary. In Fig. 4 we have plotted $\sigma$ 's for binaries all located at the same distance as a function of total mass. We fix one of the masses at a value of $1.4 M_{\odot}$ and vary the other from $1.4 M_{\odot}$ to $10 M_{\odot}$. In computing the $\sigma$ 's plotted in this figure we have 
terminated the waveform at the plunge orbit and normalised the SNR of a $10 M_{\odot}-1.4 M_{\odot}$ binary system to 10 . As a function of $M$ the uncertainties in $\tau_{0}$ and $\tau_{1}$ initially falloff since the increase in the SNR for larger mass binaries more than compensates for the drop in the upper frequency cutoff. However, for $M$ larger than a certain $M_{0}$ the increase in SNR is not good enough to compensate for the drop in $f_{c}$ so much so that the uncertainties in $\tau_{0}$ and $\tau_{1}$ increase beyond $M_{0}$. The parameter $t_{a}$, however, falls off monotonically.

\section{B. Monte Carlo estimation of parameters}

In this Section we present the first in a series of efforts to compute the covariance matrix of errors through numerical simulations for a coalescing binary waveform at various postNewtonian orders. Analytical computation of the covariance matrix, as in the previous section, gives us an idea of the covariances and variances but, as we shall see in this Section, at low SNR's it grossly underestimates the errors. Quite apart from the fact that the assumptions made in deriving the covariance matrix might be invalid at low SNR's, in a realistic detection and data analysis, other problems, such as discreteness of the lattice of templates, finite sampling of the data, etc., do occur. It therefore seems necessary to check the analytical calculations using numerical simulations to gain further insight into the accuracy at which physical parameters can be measured. This Section is divided into several parts: In the first part we highlight different aspects of the simulation, in the second part we briefly discuss the choice of templates for the simulation, in the third we elaborate on the Monte Carlo method that we have adopted to carry out our simulations and in the fourth we discuss problems that arise in a numerical simulation. The results of our study are discussed in the next Section.

\section{Parameters of the simulation}

Let $s(t)$ be a signal of strength $\mathcal{A}$ characterised by a set of parameters $\hat{\boldsymbol{\lambda}}$

$$
s(t ; \hat{\boldsymbol{\lambda}})=\mathcal{A} h(t ; \hat{\boldsymbol{\lambda}}), \quad\langle h, h\rangle=1 .
$$

In data analysis problems one considers a discrete version $\left\{s^{k} \mid k=0, \ldots, N-1\right\}$ of the waveform $s(t)$ sampled at uniform intervals in $t$ :

$$
s^{k} \equiv s(k \Delta) ; \quad k=0, \ldots, N-1,
$$

where $\Delta$ denotes the constant interval between consecutive samples and $N$ is the total number of samples. The sampled output $x^{k}$ of the detector consists of the samples of the noise plus the signal:

$$
x^{k}=n^{k}+s^{k} .
$$

The sampling rate, $f_{s}=\Delta^{-1}$ (also referred to as the sampling frequency) is the number of samples per unit time interval. In a data analysis problem the sampling frequency is determined by the signal bandwidth. If $B$ is the signal bandwidth, i.e., if the Fourier transform of the signal is only nonzero over a certain interval $B$, then it is sufficient to sample at a rate $f_{s}=2 B$. In our case there is a lower limit in the frequency response of the detector since the detector noise gets very large below a seismic cutoff at about 10-40 Hz. As mentioned in the last Section there is also an upper limit in frequency up to which a chirp signal is tracked since one does not accurately know the waveform beyond the last stable orbit of the binary. This corresponds to gravitational wave frequency $f_{c}=1 /\left(6^{3 / 2} \pi M\right)$. For a neutron star-neutron star (ns-ns) binary $f_{c} \sim 1525 \mathrm{~Hz}$ while for a ns-black hole (of $10 M_{\odot}$ ) (ns-bh) binary $f_{c} \sim 375 \mathrm{~Hz}$. Due to constraints arising out of limited computational power, we terminate waveforms at $750 \mathrm{~Hz}$ even when $f_{c}$ is larger than $1000 \mathrm{~Hz}$. Such a shutoff is not expected to cause any spurious results since, even in the case of least massive binaries of ns-ns, which we consider in this study, more than $99 \%$ of the 'energy' is extracted by the time the signal reaches $750 \mathrm{~Hz}$. We have carried out simulations with two types of upper cutoff: 
1. one in which all templates, irrespective of their total mass, are shutoff beyond $750 \mathrm{~Hz}$,

2. a second in which the upper frequency cutoff is chosen to be $750 \mathrm{~Hz}$ or $f_{c}$, whichever is lower.

Consistent with these cutoffs the sampling rate is always taken to be $2 \mathrm{kHz}$. (We have carried out simulations with higher sampling rates and found no particular advantage in doing so nor did we find appreciable changes in our results.)

In all our simulations, as in the previous section, we take the detector noise power spectral density $S$ to be that corresponding to initial LIGO 21]. For the purpose of simulations we need to generate noise corresponding to such a power spectrum. This is achieved by the following three steps:

1. generate Gaussian white noise $n^{\prime k}$ with zero mean and unit variance,

$$
\overline{n^{\prime k}}=0, \overline{n^{\prime k} n^{\prime l}}=\delta^{k l}
$$

where an overbar denotes average over an ensemble,

2. compute its Fourier transform

$$
\tilde{n}^{\prime k} \equiv \frac{1}{\sqrt{N}} \sum_{l=0}^{N-1} n^{\prime l} \exp (2 \pi i k l / N)
$$

and

3. multiply the Fourier components by the square root of the power spectral density,

$$
\tilde{n}^{k}=\sqrt{S^{k}} \tilde{n}^{\prime k}
$$

The resultant random process has the requisite power spectrum. In the above, the second step can be eliminated since the Fourier transform of a Gaussian random process is again a Gaussian, but with a different variance. In other words we generate the noise directly in the Fourier domain. The simulated detector output, in the presence of a signal $s^{k}$, in the Fourier domain is given by

$$
\tilde{x}^{k}=\tilde{n}^{k}+\tilde{s}^{k}
$$

where $\tilde{s}^{k}$ is the discrete Fourier transform of the signal.

\section{Choice of templates}

To filter a Newtonian signal we employ the set of parameters $\left\{t_{a}, \Phi, \tau_{0}\right\}$ and to filter a post-Newtonian signal we employ the set $\left\{t_{a}, \Phi, \tau_{0}, \tau_{1}\right\}$. Templates need not explicitly be constructed for the time of arrival since computation of the scalar product in the Fourier domain (and the availability of fast Fourier transform (FFT) algorithms) takes care of the time of arrival in essentially one computation $\left(N \log _{2} N\right.$ operations as opposed to $N^{2}$ operations, where $N$ is the number of data points). Moreover, there exists a two-dimensional basis for the phase parameter which allows the computation of the best correlation with the aid of just two filters. Consequently, the parameter space is essentially one-dimensional in the case of Newtonian signals and two-dimensional in the case of post-Newtonian signals. (However, as shown in Section IIIC it is to be noted that for the purpose of detection the effective dimensionality of the parameter space, even with the inclusion of second post-Newtonian corrections, is only one-dimensional.) We adopt the method described in Sathyaprakash and Dhurandhar 13 to determine the templates needed for chirp times. As described in 13,28 filters uniformly spaced in $\tau_{0}$ and $\tau_{1}$ covers the parameters space efficiently. 


\section{Monte Carlo method}

In order to compute variances and covariances numerically, we employ the Monte Carlo method. The basic idea here is to mimic detection and estimation on a computer by performing a very large number of simulations so as to minimize the uncertainties induced by noise fluctuations. In our simulations we generate a number of detector outputs $\left\{x^{k}\right\}$ each corresponding to a definite signal $s^{k}(\hat{\boldsymbol{\lambda}})$ of a certain strength, but corresponding to different realisations of the random process $\left\{n^{k}\right\}$. Computation of the covariance matrix involves filtering each of these detector outputs through an a priori chosen set (or lattice) of templates $\left\{q\left(t ;{ }_{\mathrm{t}} \boldsymbol{\lambda}_{k}\right) \mid k=1, \ldots, n_{f}\right\}$, where $n_{f}$ denotes the number of templates. The templates of the lattice each has a distinct set of values of the test parameters ${ }_{\mathrm{t}} \boldsymbol{\lambda}_{k}$ and together they span a sufficiently large volume in the parameter space. The simulated detector output is correlated with each member of the lattice to obtain the corresponding filtered output $C\left({ }_{\mathrm{t}} \boldsymbol{\lambda}_{k}\right)$ :

$$
C\left({ }_{\mathrm{t}} \boldsymbol{\lambda}_{k}\right)=\left\langle x, q\left({ }_{\mathrm{t}} \boldsymbol{\lambda}_{k}\right)\right\rangle
$$

For a given realisation of noise a particular template obtains the largest correlation and its parameters are the measured values ${ }_{\mathrm{m}} \boldsymbol{\lambda}$ of the signal parameters. Thus, the measured values of the parameters are defined by

$$
\max _{k} C\left({ }_{\mathrm{t}} \boldsymbol{\lambda}_{k}\right)=C\left({ }_{\mathrm{m}} \boldsymbol{\lambda}\right)
$$

The measured values, being specific to a particular realisation of noise, are random variables. Their average provides an estimation ${ }_{\mathrm{e}} \boldsymbol{\lambda}$ of the true parameter values and their variance is a measure of the error $\sigma_{\boldsymbol{\lambda}}$ in the estimation:

$$
{ }_{\mathrm{e}} \boldsymbol{\lambda}=\overline{{ }_{\mathrm{m}} \boldsymbol{\lambda}}, \quad \sigma_{\boldsymbol{\lambda}}^{2}=\overline{\left({ }_{\mathrm{m}} \boldsymbol{\lambda}-\overline{\mathrm{m}^{\lambda} \boldsymbol{\lambda}}\right)^{2}}, \quad D^{\mu \nu}=\frac{\overline{\mathrm{m}^{\lambda^{\mu}{ }_{\mathrm{m}} \lambda^{\nu}}}}{\sigma_{\mu} \sigma_{\nu}}, \quad(\mu \neq \nu),
$$

where $D_{\mu \nu}$ are the correlation coefficients between parameters $\lambda_{\mu}$ and $\lambda_{\nu}$. In order to accurately determine $\sigma_{\boldsymbol{\lambda}}$ a large number of simulations would be needed. If the measured values ${ }_{\mathrm{m}} \boldsymbol{\lambda}$ obey Gaussian statistics then after $N_{\mathrm{t}}$ trials the variance is determined to a relative accuracy $1 / \sqrt{N}_{\mathrm{t}}$ and estimated values can differ from their true values by $\sigma_{\boldsymbol{\lambda}} / \sqrt{N}_{\mathrm{t}}$. We have performed in excess of 5000 trials, for each input signal, and thus our results are accurate to better than 1 part in 70 . Even more crucial than the number of simulations is the number of templates used and their range in the parameter space. We discuss these and other related issues next.

The actual templates chosen, say for the parameter $\tau_{0}$, in a given 'experiment' depend on the true parameters of the signal, the number of noise realisations employed and the expected value of the error. Let us suppose we have a first guess of the error in $\tau_{0}$, say $\sigma_{\tau_{0}}$. Then, we choose 51 uniformly spaced filters around $\hat{\tau}_{0}$ (where $\hat{\tau_{0}}$ is the signal chirp time) such that:

$$
{ }_{\mathrm{t}} \tau_{0} \in\left[\hat{\tau}_{0}-5 \sigma_{\tau_{0}}, \hat{\tau}_{0}+5 \sigma_{\tau_{0}}\right]
$$

This implies that we are covering a $5 \sigma$ width in $\tau_{0}$ at a resolution of $\sigma_{\tau} / 5$. The probability, that a template between $4 \sigma$ and $5 \sigma$ from the true signal 'clicks', being $\sim 6 \times 10^{-5}$, we are on safe grounds since, in a given simulation, we consider no more than 5000 trials. (In comparison, the probability that a template between $3 \sigma$ and $4 \sigma$ clicks is $2.2 \times 10^{-3}$ corresponding to an expected 13 events in 5000 trials.) For a post-Newtonian signal, which in effect needs to be spanned by a two-dimensional lattice of filters, the above choice of templates implies a requirement of $2601 \times 2$ filters in all. Here a factor of 2 arises because for each filter in the $\tau_{0}-\tau_{1}$ space we will need two templates corresponding to the two independent values of the phase $\Phi: 0$ and $\pi / 2$. In the case of a Newtonian signal, the lattice being one-dimensional, one can afford a much higher resolution and range. Even with the aid of a mere 201 templates we can probe at a $\sigma / 10$ resolution with a $10 \sigma$ range.

We start off a simulation with the pretension that there is no knowledge of what the $\sigma_{\boldsymbol{\lambda}}$ 's are. Thus, we choose as our first trial a very large $\sigma_{\boldsymbol{\lambda}}$ and lay the lattice of templates. 
With this lattice we perform a test run of 400 trials and examine the distribution of the measured values. If the distribution is not, as expected, a Gaussian then we alter $\sigma_{\boldsymbol{\lambda}}$ : we decrease it if the distribution is too narrow and increase it if the distribution is too wide and does not show the expected falloff. In particular, we make sure that the templates at the boundary of the chosen range do not click even once and the skewness of the distribution is negligible. When for a certain $\sigma_{\boldsymbol{\lambda}}$ a rough Gaussian distribution is observed then we carry out a simulation with a larger number of trials (typically 5000). We subject the measured values in this larger simulation to the very same tests described above. We only consider for further analysis such simulations which 'pass' the above tests and determine the estimates, variances and covariances of the parameters using the measured values, with the aid of equation (4.13).

\section{Numerical errors and remedies}

There are several sources of numerical errors that tend to bias the results of a simulation unless proper care is exercised to rectify them. In this Section we point out the most important ones and show how they can be taken care of. Due to memory restrictions, the present version of our codes work with single precision except the FFT, which is implemented in double precision. In future implementations we plan to carry out all computations in double precision. This will possibly reduce some of the numerical noise that occurs, especially at high SNRs, in the present simulations.

1. Orthonormality of filters: For the sake of simplicity it is essential that the filters are normalised in the sense that their scalar product is equal to unity: $\langle q, q\rangle=1$. A waveform is normalized numerically using the discrete version of the scalar product:

$$
\mathcal{N}=\frac{1}{\sum_{k=0}^{N-1} S_{k}^{-1}\left|\tilde{q}^{k}\right|^{2}}
$$

As mentioned earlier we use a two-dimensional basis of filters for the phase parameter. Choosing the two filters to be orthogonal to each other makes the maximisation over phase easier. However, here care must be exercised. Two filters $q\left(t ; t_{a}, \tau_{0}, \tau_{1}, \Phi=0\right)$ and $q\left(t ; t_{a}, \tau_{0}, \tau_{1}, \Phi=\pi / 2\right)$ are apparently orthogonal to each other. The numerically computed 'angle' between the two filters, chosen in this manner, often turns out to be greater than $\sim 10^{-2}$ radians. Consequently, one obtains erroneous correlations. In order to circumvent this problem we first generate two filters that are roughly orthogonal to each other, as above, and then use the Gram-Schmidt method to orthogonalise the two vectors. If an explicit numerical orthogonalisation such as this is not implemented then the measured values of the various parameters show spurious oscillations in their distribution and the estimated values of the parameters tend to get biased.

2. Correlation function: The scalar product of two normalised templates $q\left(t ;{ }_{t} \boldsymbol{\lambda}\right)$ and $q\left(t ;{ }_{\mathrm{t}} \boldsymbol{\lambda}^{\prime}\right)$ is given by

$$
C\left({ }_{\mathrm{t}} \lambda_{\mu},{ }_{\mathrm{t}} \lambda_{\nu}^{\prime}\right)=\left\langle q\left(t ;{ }_{\mathrm{t}} \lambda_{\mu}\right), q\left(t ;{ }_{\mathrm{t}} \lambda_{\nu}^{\prime}\right)\right\rangle, \quad\left\langle q\left(t ;{ }_{\mathrm{t}} \boldsymbol{\lambda}\right), q\left(t ;{ }_{\mathrm{t}} \boldsymbol{\lambda}\right)\right\rangle=\left\langle q\left(t ;{ }_{\mathrm{t}} \boldsymbol{\lambda}^{\prime}\right), q\left(t ;{ }_{\mathrm{t}} \boldsymbol{\lambda}^{\prime}\right)\right\rangle=1,
$$

where we have indicated the dependence of the scalar product on the various parameters by explicitly writing down the parameter subscripts. Let us fix the parameters of one of the templates, say ${ }_{\mathrm{t}} \boldsymbol{\lambda}$, and vary the parameters of the other template. Of particular interest is the behaviour of $C$ maximised over all but one of the parameters, say $\lambda_{\nu_{0}}$ :

$$
C_{\max }\left({ }_{\mathrm{t}} \lambda_{\mu},{ }_{\mathrm{t}} \lambda_{\nu_{0}}^{\prime}\right)=\max _{\substack{\mathrm{t} \lambda_{\nu}^{\prime} \\ \nu \neq \nu_{0}}} C\left({ }_{\mathrm{t}} \lambda_{\mu},{ }_{\mathrm{t}} \lambda_{\nu}^{\prime}\right)
$$


$C_{\max }$ is expected to drop monotonically as $\left|\lambda_{\nu_{0}}-\lambda_{\nu_{0}}^{\prime}\right|$ increases. However, we have observed departures from such a behaviour possibly arising out of numerical noise. Such a behaviour causes bias in the estimation of parameters, and consequently in the determination of their covariances, especially at high SNRs. We have found no remedy to this problem and some of our results at high SNRs may have biases introduced by this effect. (Sampling the templates at a higher rate did not help in curing this problem.)

3. Grid effects: The parameters of a signal chosen for the purpose of simulation and detection can in principle be anything and in particular it need not correspond to any of the templates of the lattice. However, in practice we find that whenever the signal parameters do not correspond to a member of the lattice then the resultant simulation has a bimodal distribution of the measured values. This is, of course, expected since a signal not on the grid is picked up by two nearest templates along each direction in the parameter space. Sometimes we do find that the peaks corresponding to the bimodal distribution does not belong to the nearest neighbour filters but slightly away. This is related to the fact that the correlation function maximised over the time of arrival and the phase of the signal falls off much too slowly along the $\tau_{0}-\tau_{1}$ direction and small deviation from a monotonic fall can cause biases. (Such biases would be present in the case when a signal corresponds to one of the grid points though the magnitude of the effect would be lower.) In order to avoid this problem, and the consequent shifts in the estimation of parameters and errors in the determination of variances and covariances, we always choose the parameters of the signal to be that corresponding to a template.

\section{Upper frequency cutoff and its effect on parameter estimation}

The Fisher information matrix computed using the stationary phase approximation in Section III does not include the effect of truncating the waveform at $a=6 \mathrm{M}$ - the plunge cutoff. As mentioned before, we have carried out simulations for both with, and without, incorporating the upper cutoff. As the covariance matrix incorporating the upper cutoff is not available we have been able to compare the Monte Carlo results with the covariance matrix only for the latter case, where the cutoff is held fixed at $750 \mathrm{~Hz}$. If we incorporate the upper cutoff into the Monte Carlo simulations the errors in the parameters are reduced drastically. The effect of the upper cutoff is expected to be more important for the higher mass binaries such as the ones we have considered. The ambiguity function, in this case, no longer remains independent of the point on the manifold. In other words, the correlation between two chirps depends not only on the difference between the parameters of the signals, but also on the absolute values of the parameters. The correlation surface also ceases to be symmetric i.e. the correlation between two chirps also depends on the sign of $\delta \lambda$, where, $\delta \lambda$ is the difference in the values of the parameters. As the computational power required for carrying out simulations for lower mass binaries is not available to us the simulations have been restricted to ns-bh star binaries, where the effect of the upper cutoff is important.

\section{Boundary effects}

For the purpose of simulations a grid of filters has to be set up 'around' the signal. The grid must be large enough so that the estimated parameters do not overshoot the boundary of the grid. This causes a problem as every value in the $\left\{\tau_{0}, \tau_{1}\right\}$ plane does not lead to a meaningful value for the masses of the binary system. This does not however prevent us to construct a waveform with such a value for $\left\{\tau_{0}, \tau_{1}\right\}$ even though the signal in general does not correspond to any 'real' binary system. This is valid, and even necessary, if we are to compare the numerical results with the covariance matrix.

\section{Incorporating the cutoff in the presence of boundary effects}

If we wish to incorporate the effects of the upper cutoff in simulations then we run into a serious problem, as we would have to know the total mass of the binary in order to compute the upper cutoff. For an arbitrary $\left\{\tau_{0}, \tau_{1}\right\}$ we can end up with negative and even complex values of the total mass and hence the upper cutoff at $a=6 \mathrm{M}$ is not 
meaningful. Thus, we cannot even construct a waveform for an arbitrary combination of $\left\{\tau_{0}, \tau_{1}\right\}$. Therefore, in such cases, we restrict ourselves to simulations where the grid lies entirely within valid limits for $\left\{\tau_{0}, \tau_{1}\right\}$.

\section{Results and Discussion}

Our primary objective is to measure the variances and covariances following the method described in Sec. IV B 3 and study their departure from that predicted by analytical means (cf. Sec. IVA). We have carried out simulations for several values of the masses of the binary and in each case the signal strength (which is a measure of the SNR) is varied in the range 10-40. However, since the variances and covariances are independent of the absolute values of the parameters, for the parameter set that we employ, results are only quoted corresponding to a typical binary system. (See Sec. IV A for a discussion of the covariance matrix.) Similar results are obtained in other cases too. We use two sets of parameters to describe our results. Monte Carlo simulations allow us to directly measure the amplitude, the time of arrival, the phase at the time of arrival and the chirp time(s). This is the set $\left\{\mathcal{A}, t_{a}, \Phi, \tau_{0}, \tau_{1}\right\}$.

As we shall see below, the instant of coalescence can be measured much more accurately than the time of arrival. As a consequence of this, the direction to the source can be determined at a much greater accuracy by employing $t_{C}$ as a parameter instead of $t_{a}$. Thus, we also quote estimates and errors for the parameter $t_{C}$. Since the error in the estimation of the phase is quite large, even at high SNRs, we ignore it in our discussions.

We first deal with the Newtonian signal and highlight different aspects of the simulation and discuss the results at length. We then consider the first post-Newtonian corrected signal.

\section{Newtonian signal}

In the case of Newtonian signals the parameter space is effectively one-dimensional and, as mentioned earlier, in this case the lattice of templates covers a $10-\sigma$ range of the parameters at a resolution of $\sigma / 10$ centred around the true parameters of the signal.

In Fig. 5 we have shown the error $\sigma_{\boldsymbol{\lambda}}$ in the estimation of parameters $t_{a}, \tau_{0}$ and $t_{C}$, as a function of SNR, deduced using the covariance matrix as solid lines and computed using Monte Carlo simulations as dotted lines. The curve corresponding to the covariance matrix is obtained using an upper frequency cutoff $f_{c}=750 \mathrm{~Hz}$ consistent with that used in our simulations. The errorbars in the estimation of $\sigma_{\boldsymbol{\lambda}}$ 's are obtained using 4 simulations, each with 4000 trials. At low SNR's $\sigma_{\boldsymbol{\lambda}}$ 's have a larger uncertainty, as expected, and for $\rho>30$ this uncertainty is negligible, and sometimes smaller than the thickness of the curves, except in the case of $\sigma_{t_{C}}$ (see below, for a possible explanation).

At low SNRs (10-15) there is a large departure of the various $\sigma_{\boldsymbol{\lambda}}$ 's from that inferred using the covariance matrix. At an SNR $\sim 17$ the two curves merge (except in the case of $\sigma_{t_{C}}$ ) indicating the validity of the covariance matrix results for this and higher SNR's. Interestingly, the agreement between Monte Carlo simulation results and those obtained using the covariance matrix is reached roughly at the same SNR irrespective of the parameter in question.

We note that in spite of the fact that the time of arrival and the chirp time have large errors, the instant coalescence can be estimated very accurately - an order of magnitude better than either. What is puzzling, however, is that, in the case of $t_{C}$, the Monte Carlo curve drops below the covariance matrix curve above an SNR of 15 and the two curves do not seem to converge to one another even at very high SNR's. Coincident with the crossover of the two curves, the error in the estimation of $\sigma_{t_{C}}$ increases, contrary to what happens for the other parameters, signalling that there is a large fluctuation in the estimation of $\sigma_{t_{C}}$. This behaviour, we guess, is an artifact of the low value of the sampling rate. Of course, our sampling rate is sufficiently high to respect the sampling theorem. However, since $t_{C}$ is determined to an accuracy an order of magnitude better than either $t_{a}$ or $\tau_{0}$, a much higher resolution in template-spacing would be needed for determining the error in the instant 
of coalescence than that used for estimating the errors in the time of arrival or the chirp time(s). Testing this claim, unfortunately, is beyond the computer resources at our disposal since we would need a sampling rate of about $10 \mathrm{kHz}$ with a filter-spacing $10^{-4} \mathrm{~s}$. We hope to be able to resolve this issue in course of time. Nevertheless, the fact that the error in the estimation of $\sigma_{t_{C}}$ first decreases with SNR and increases only after the two curves crossover, hints at the above possibility as a cause for this anomalous behaviour. This effect is also observed in the case of a post-Newtonian signal.

In Table $\bigvee$ we have given the actual signal parameters $\hat{\boldsymbol{\lambda}}$, estimated values of the parameters ${ }_{\mathrm{e}} \boldsymbol{\lambda}$ (cf. equation (4.13)) and the corresponding errors in their estimation $\sigma_{\boldsymbol{\lambda}}$, for several values of the SNR. Errors inferred from the covariance matrix can be read off from Fig. 5 . The estimated values are different from the true values, some of them being overestimated and some others being underestimated. However, the deviations are often larger than what we expect. In a simulation that uses $N_{\mathrm{t}}$ trials the estimated parameters ${ }_{\mathrm{e}} \boldsymbol{\lambda}$, assuming a Gaussian distribution for the measured parameters ${ }_{\mathrm{m}} \boldsymbol{\lambda}$, can be different from the true values by $\sigma_{\boldsymbol{\lambda}} / \sqrt{N_{\mathrm{t}}}$. (In contrast, the measured values ${ }_{\mathrm{m}} \boldsymbol{\lambda}$ can differ from their true values by $\sigma_{\boldsymbol{\lambda}}$ or more.) However, we often obtain a slightly larger deviation,

$$
2 \frac{\sigma_{\boldsymbol{\lambda}}}{\sqrt{N_{\mathrm{t}}}} \lesssim\left|{ }_{\mathrm{e}} \boldsymbol{\lambda}-\hat{\boldsymbol{\lambda}}\right| \lesssim 3 \frac{\sigma_{\boldsymbol{\lambda}}}{\sqrt{N_{\mathrm{t}}}},
$$

and we are unable to resolve this discrepancy. A more concrete test for the simulations is the histogram $n\left({ }_{\mathrm{m}} \boldsymbol{\lambda}\right)$ of the measured parameters, namely the frequency at which a given parameter clicks in a simulation. This is shown plotted in Fig. 6 for an SNR of 10. The skewness of the measured value is less than $10^{-2}$. These results lend further support to the Monte Carlo simulations. There are visible asymmetries in the distributions of $\tau_{0}$ and $t_{a}$ and the asymmetries in the two cases are of opposite sense. This can, of course, be understood from the fact that $t_{a}$ and $\tau_{0}$ have a negative correlation coefficient. The histogram of $t_{C}$, even at an SNR of 10, has very few non-zero bins. This of course reflects the fact that it is determined very accurately. We are unable to resolve the central peak in $n\left(t_{C}\right)$ since, as mentioned earlier, the sampling rate and resolution in $\tau_{0}$ are not good enough to do so.

\section{Post-Newtonian signal}

As opposed to the Newtonian case here we have essentially a two-dimensional lattice of filters corresponding to $\tau_{0}$ and $\tau_{1}$. For the purpose estimating variances and covariances we lay a mesh consisting of $2601 \times 2$ uniformly spaced filters around the true parameters of the signal. As pointed out in Sec. IIIB not all filters in the mesh, unlike in the Newtonian case, would correspond to the waveform from a realistic binary but that does not preclude their use in the Monte Carlo simulations. We shall see that the results of our simulations lend further support to the claim that for the purpose of detection, the parameter space need only be onedimensional [28]. The results obtained for the first post-Newtonian signal are qualitatively similar to that of a Newtonian signal and we refer the reader, where appropriate, to the Newtonian case for a more complete discussion.

In Fig. 7 we have shown the error in the estimation of parameters $\tau_{0}, \tau_{1}, t_{C}$ and $t_{a}$, clockwise from top left, respectively, as a function of SNR. The solid and dotted curves are as in Fig. 5. Here again the upper frequency cutoff is taken to be $750 \mathrm{kHz}$. Just as in the case of a Newtonian signal here too the results obtained from Monte Carlo simulation are much higher than those obtained by employing the covariance matrix. At an SNR of 10, which is the expected value for a majority of events that initial LIGO and VIRGO interferometers will observe, the Monte Carlo values are more than thrice as much as their corresponding covariance matrix values and at an SNR of 15 the errors are roughly twice that expected from the covariance matrix. In absolute terms, however, the errors are still quite small compared to the actual parameter values: for a ns-ns binary, at an SNR of 10,

$$
\frac{\sigma_{\tau_{0}}}{\tau_{0}} \sim 2.4 \%, \quad \frac{\sigma_{\tau_{1}}}{\tau_{1}} \sim 9.4 \% .
$$

At an SNR of 10 the time of arrival can be measured to an accuracy of $72 \mathrm{~ms}$ in contrast to a value of $20 \mathrm{~ms}$ expected from the covariance matrix. As is well known, with the inclusion 
of the post-Newtonian terms error in the estimation of the time of arrival and Newtonian chirp time increases by about a factor of 2 and 3, respectively [21,22].

As in the Newtonian case here again we see that the Monte Carlo curves approach the corresponding covariance matrix curves at a high SNR the only difference being that the agreement is reached at a higher SNR $\sim 25$. For SNRs larger than this the two curves are in perfect agreement with each other. As mentioned earlier, $\sigma_{t_{C}}$ shows an anomalous behaviour possibly arising out of insufficient resolution in the time of arrival and the chirp times.

In Table $\mathbb{V}$ we have listed the true parameters $\hat{\boldsymbol{\lambda}}$, the estimated values ${ }_{\mathrm{e}} \boldsymbol{\lambda}$ and the Monte Carlo errors $\sigma_{\boldsymbol{\lambda}}$ for different SNRs. As in the Newtonian case here too the estimated values show a larger departure, than expected, from the true values. Histograms of the various measured parameters including $t_{C}$ are shown in Fig. 8 for a signal strength of 10 . The skewness is below its standard deviation of $\sqrt{15 / N_{\mathrm{t}}}$ 33 indicating the Gaussian nature of the various distributions. Even in the case of a post-Newtonian signal $\sigma_{t_{C}}$ is so small that we only have three non-zero bins in $n\left(t_{C}\right)$.

We now turn attention to other, more general, issues arising out of the simulations.

In Sec. III C we have argued, on the basis of the behaviour of the noise-free correlation function, that the effective dimensionality of the parameter space for the purpose of detection, even in the case of a post-Newtonian signal, is only one-dimensional. The results of our Monte Carlo simulation unambiguously show that this is indeed true even in the presence of noise. We investigated the two-dimensional histogram, that gives the number of occurrances of different templates in the lattice, in a particular simulation. The templates that 'click' are all aligned along the line $\tau_{0}+\tau_{1}=$ const. In a total of 5000 realisations corresponding to this simulation there is only one instance when a filter outside this region clicks giving a probability of less than $10^{-3}$ for a template outside this region to give a maximum. Consequently, it is only necessary to choose a single filter along this curve.

The distribution of the maximum correlation $C_{\max }\left(\hat{\boldsymbol{\lambda}},{ }_{t} \boldsymbol{\lambda}\right)$ obtained from different noise realisations needs a special mention since it has an inherent bias. In Fig. 9 we have shown the distribution of the maximum correlation taken from one of our simulations corresponding to an SNR of 10. Notice a slight shift of the distribution towards a higher value and this cannot be accommodated within the expected fluctuation in the mean. The measured value of the standard deviation $\sigma_{\mathcal{A}}$ is 0.95 . Since the number of simulations is 5000 we expect that the signal strength should differ from the true value of 10 by no more than $\sigma_{\mathcal{A}} / \sqrt{5000}=0.014$. However, the mean value is 10.26 giving a deviation of 0.26 which is about 20 times larger than that expected. This occurs at all SNRs and for both Newtonian and post-Newtonian signals. This of course does not mean there is bug in the way we are computing the maximum correlation. In the process of maximisation, values greater than the signal strength are favoured and consequently the mean of the maximum correlation shows a shift towards a higher value. This suggests that that the maximum of the correlation is a biased estimator of the signal strength. We find, consistent with the covariance matrix calculation, that the amplitude parameter is uncorrelated with the rest of the parameters; crosscorrealtion coefficients $D_{0} \mu, \mu \neq 0$, (cf. eq. 4.2) inferred from our Monte Carlo simulations are less than $\sim 10^{-6}$.

Finally, it is of interest to note how the phase parameter $\Phi$ is correlated with the time of arrival. A plot of ${ }_{\mathrm{m}} \Phi$ versus ${ }_{\mathrm{m}} t_{a}$ is shown in Fig. 10. We find that the measured values of the time of arrival and the phase are such that $2 \pi f_{0} t_{m} t_{a}={ }_{\mathrm{m}} \Phi$, where $f_{0}$ has a value of approximately $51 \mathrm{~Hz}$. When the time of arrival shifts by more than a cycle of the signal the phase jumps by a factor $2 \pi$ leading to the points seen in the top-left and bottom-right corner of the figure. This makes the estimation of the phase and the error in its estimation pretty involved.

\section{Incorporating the effects of upper cutoff}

As mentioned before, incorporating an upper cutoff at the onset of the plunge has a drastic effect on the estimation of parameters. The incorporation of the upper cutoff is implemented by stopping the waveform when the instantaneous frequency reaches the fre- 
quency associated with the onset of the plunge or $750 \mathrm{~Hz}$, whichever is lower. However, due to computational constraints we have carried out the simulations only for high mass binaries and hence the upper cutoff plays an important role in all our simulations. It is to be noted that the discussion of the ambiguity function in section III C is not valid when the effect of the upper cutoff is taken into account though for low mass binaries, such as ns-ns binaries, the results there will still be valid. The further dependence of the signal waveform on the total mass of the system through the upper cutoff means that we can estimate the individual masses more exactly though the computational power is bound to increase.

In order to carry out the simulations for the present case we selected a $10 M_{\odot}-1.2 M_{\odot}$ binary system as this enables us to choose the filter grid well within valid limits of $\tau_{0}$ and $\tau_{1}$. The simulations were carried out for various values of SNR starting from 10 . The histograms of the estimated parameters at an SNR of 10 is shown in fig. 11. At this SNR the errors obtained are $\sigma_{\tau_{0}}=39.3 \mathrm{~ms}, \sigma_{\tau_{1}}=22.4 \mathrm{~ms}, \sigma_{t_{a}}=23.1 \mathrm{~ms}$ and $\sigma_{t_{C}}=0.6 \mathrm{~ms}$. These can be compared with the values in table $\mathrm{V}$ and we can see that except for the parameter $t_{C}$ the errors are substantially lesser when the upper cutoff is incorporated into the waveform. It is necessary to recompute the covariance matrix, as emphasized before, including the effect of the upper cutoff in order to compare these numerically obtained values with the covariance matrix. In order to do this it is not enough to replace the upper limit in the integral in eq. (3.19) with the upper cutoff. The waveform now depends on the total mass of the system through the upper cutoff and this information has to be incorporated into the waveform.

We carried out the simulations for various SNRs for the same value of masses quoted above. In the absence of the estimates of the covariance matrix when the upper cutoff is incorporated we assume that at an SNR of 40 the Monte Carlo estimates are consistent with those of the covariance matrix. In Figure 12 we illustrate the results of our simulations. The points on the dotted line are the values obtained through Monte Carlo estimates and the continuous line is obtained by fitting a $1 / \rho$ dependence of the errors on the SNR assuming consistency at an SNR of 40. It is seen as in the previous simulations that except for the parameter $t_{C}$ the other parameters are fairly consistent with a $1 / \rho$ dependence of the errors on the SNR at an SNR greater than 25.

\section{CONCLUSIONS}

In this paper we have introduced the use of differential geometry in studying signal analysis and have addressed issues pertaining to optimal detection strategies of the chirp waveform. We have also carried out Monte Carlo simulations to check how well the covariance matrix estimates the errors in the parameters of the chirp waveform. We summarize below our main results.

1. We have developed the concept of a signal manifold as a subset of a finite dimensional vector space of detector outputs. Using the correlation between two signal vectors as a scalar product we have induced a metric on the signal manifold. With this geometric picture it is possible to pose the question of optimal detection in a more general setting. We suggest that the set of template waveforms for the detection of the chirp signal need not correspond to any point on the chirp manifold. We propose an algorithm to choose templates off the signal manifold and show that the drop in the correlation due to the discreteness of the set of templates is reduced. This algorithm, though certainly not the best, motivates the search of more efficient templates. In addition, the chirp manifold corresponding to the second post-Newtonian waveform is shown to be effectively one-dimensional. This has important implications for the computational requirement for the on-line detection of the chirp signal. The use of a convenient set of parameters of the chirp waveform for carrying out numerical and analytical simulations is stressed. These parameters are such that the metric components are independent of the parameters which implies that the manifold is flat and the corresponding 'coordinate system' is Cartesian. As the metric defined is nothing but the Fisher information matrix, the covariance matrix, being the inverse of the Fisher information matrix, is also independent of the parameters. 
2. Monte Carlo simulations have been carried out for the case of the initial LIGO to find out whether the actual errors in the estimation of parameters is consistent with the values predicted by the covariance matrix. Simulations have been carried out for both the Newtonian as well as the post-Newtonian waveforms. We have restricted ourselves to the case of high mass binary systems, such as bh-ns binaries, where the computational requirement is not very heavy since the length of the data train, in such cases, works out to be less than $8 \mathrm{~s}$. Nevertheless, as has been shown in this paper, the covariance matrix is independent of the parameters identified by us when waveforms are terminated at a constant upper cutoff irrespective of their masses. Consequently, our results will hold good for binary systems of arbitrary masses. We point out the major problems that arise while performing a numerical simulation and, where appropriate, we suggest how they may be taken care of. In particular, the effect of incorporating the upper cutoff in the frequency of the gravitational wave at the onset of the plunge, which essentially depends on the total mass of the binary, is extremely important for high mass binaries. Since the covariance matrix with the inclusion of such a mass-dependent upper cutoff is not available we have carried out most of our simulations using a constant upper cutoff. This enables us to directly compare the results of our Monte Carlo simulations with those of the analytically computed covariance matrix. Since for binaries with total mass less than $5 M_{\odot}$ the plunge induced upper cutoff is larger than that induced by the detector noise these effects can be ignored for such binaries.

The numerical experiments indicate that the covariance matrix underestimates the errors in the determination of the parameters even at SNRs as high as 20. In the Newtonian case the correlation coefficient of the time of arrival $t_{a}$ and the Newtonian chirp time $\tau_{0}$ is found to be very close to -1 , so much so that even at a SNR of 7.5, the instant of coalescence $t_{C}=t_{a}+\tau_{0}$ remains practically a constant. The error in the estimation of $\tau_{0}$ for the post-Newtonian waveform is about four times the error obtained in the case of the Newtonian waveform at the same SNR. This is expected as the first post-Newtonian correction to the waveform introduces a new parameter $\tau_{1}$ (called the first post-Newtonian chirp time) which is highly (anti)correlated with $\tau_{0}$.

For the post-Newtonian waveform at an SNR of 10 the error in $\tau_{0}$ is about 3 times that predicted by the covariance matrix. This corresponds to a factor of 2 in the chirp mass $\mathcal{M}$. The distributions for the parameters have been obtained and are seen to be unimodal distributions and are slightly more sharper than a Gaussian. When the plunge induced upper cutoff is incorporated into the waveform the errors in the estimation of parameters decrease by a factor of about 2.5. The correlation coefficient between $\tau_{0}$ and $\tau_{1}$ is also found to decrease, which is consistent with our discussion in Section IVA.

The results obtained suggest that higher moments than that used in obtained in computing the covariance matrix may be important in the determination of the errors in parameter estimation. In the geometric picture this amounts to taking into account curvature effects, either intrinsic or extrinsic.

3. We suggest that $t_{C}$ is a more suitable parameter to estimate the direction to the source than the time of arrival. The latter is a kinematical parameter that fixes the time at which the gravitational wave frequency reaches the lower cutoff of the detector while the parameter $t_{C}$ has the physical significance of being the instant of coalescence. At an SNR of 10 the error in $t_{a}$ is too large $(20 \mathrm{~ms})$ to deduce the direction to the source accurately, whereas the error in the parameter $t_{C}$ is less than $0.5 \mathrm{~ms}$. This will further go down substantially for the advanced LIGO. A detailed analysis of the determination of the direction to the binary using delays in $t_{C}$ between different detectors is carried out in Bhawal and Dhurandhar [35] (also see [29]).

We now suggest further work which needs to be done along the lines of this paper. A full understanding of the chirp signal manifold when higher post-Newtonian corrections are incorporated into the waveform is in order. This will help in the development of more efficient algorithms for the choice of templates in the detection problem and facilitate reduction in 
computational time. The Monte Carlo simulations which we have carried our are for the case of a binary waveform correct up to first post-Newtonian order. Moreover, only circular orbits are considered. The effect of eccentricity is currently being investigated [36]. Performing simulations when higher post-Newtonian corrections are taken into account calls for an immense amount of computational time. Fortunately, matched filtering algorithm being amenable to parallelization [37], one could aim at using the massively parallel computers, that are now becoming available the world over, in performing such simulations.

\section{ACKNOWLEDGMENTS}

The authors would like to thank the members of the gravitational wave group at IUCAA especially S.D. Mohanty and B. Bhawal for many helpful discussions. R.B. is being supported by the Senior Research Fellowship of CSIR, India.

[1] A. Abramovici et. al., Science 256325 (1992).

[2] C. Bradaschia et. al., Nucl. Instrum. Methods Phys. Res., Sect A 518 (1990).

[3] B.F. Schutz, in Gravitational Collapse and Relativity, Edited by H. Sato and T. Nakamura, (World Scientific, Singapore, 1986), pp. 350-368.

[4] D. Marković, Phys. Rev. D 48, 4738 (1993).

[5] L. Blanchet and B.S. Sathyaprakash, Phys. Rev. Lett., 74, 1067 (1995).

[6] K.S. Thorne, Gravitational waves from compact objects, to be published in Proceedings of IAU symposium 165, Compact stars in binaries, edited by J. van Paradijs, E. van den Heuvel, and E. Kuulkers, (Kluwer Academic Publishers).

[7] K.S. Thorne, Gravitational waves, to be published in Proceedings of the Snowmass 95 Summer Study on Particle and Nuclear Astrophysics and Cosmology, edited by E.W. Kolb and R. Peccei, (World Scientific, Singapore).

[8] S.D. Mohanty and B.S Sathyaprakash, A modified periodogram for the detection of gravitational waves from coalescing binaries, submitted to Phys. Rev. D (1995).

[9] S, Smith, Phys. Rev. D 36, 2901 (1987).

[10] K.S. Thorne, in 300 Years of Gravitation, S.W. Hawking and W.Israel (eds.), (Cambridge Univ. Press, 1987).

[11] C.W. Helstrom, Statistical Theory of Signal Detection, 2nd. ed, (Pergamon Press, London, 1968).

[12] B.F. Schutz, in The Detection of Gravitational Radiation, edited by D. Blair (Cambridge, 1989) pp 406-427.

[13] B.S. Sathyaprakash and S.V. Dhurandhar, Phys. Rev. D 44, 3819 (1991).

[14] S.V. Dhurandhar and B.S. Sathyaprakash, Phys. Rev. D 49, 1707 (1994).

[15] R. Balasubramanian and S.V. Dhurandhar, Phys. Rev. D 50, 6080 (1994).

[16] K. Kokkotas, A. Krolak, and G. Tsegas, Class. Quant. Grav., 11, 1901 (1994).

[17] T. A. Apostolatos, Phys. Rev. D, 52, 605 (1995).

[18] A. Krolak, K.D. Kokkotas and G. Schäfer, Phys. Rev. D. in press.

[19] Shun-ichi Amari, Differential Geometric Methods in Statistics, (Springer-Verlag).

[20] L. S. Finn, Phys. Rev. D 46, 5236 (1992).

[21] L.S. Finn and D.F. Chernoff, Phys. Rev. D 47, 2198 (1993).

[22] C. Cutler and E. Flanagan, Phys.Rev. D 49, 2658 (1994).

[23] L. Blanchet and B.S. Sathyaprakash, Class. Quantum Grav., 11, 2807 (1994).

[24] A. Krolak, in Gravitational wave data analysis, edited by B.F. Schutz, (Dordrecht : Kluwer, 1989), pp. 59-69.

[25] A. Krolak, J. A. Lobo and B. J. Meers, Phys. Rev D 48, 3451 (1993).

[26] E. Poisson and C.M. Will, Phys. Rev. D 52, 848 (1995).

[27] P. Jaranowski, K.D. Kokkotas, A. Krolak and G. Tsegas, report (unpublished).

[28] B.S. Sathyaprakash, Phys. Rev. D 50, R7111 (1994). 
[29] R. Balasubramanian, B.S. Sathyaprakash and S.V. Dhurandhar, submitted for publication to, Pramana J. Phys. (1995).

[30] C. Cutler et al, Phys. Rev. Lett. 70, 2984 (1993).

[31] L. Blanchet, T. Damour and B.R. Iyer, Phys. Rev. D 51, 5360 (1995).

[32] S. V. Dhurandhar and B.F. Schutz, Phys. Rev. D 50, 2390 (1994)

[33] William H. Press, Saul A. Teukolsky, William T. Vellerling and Brian P. Flannery Numerical Recipes (Cambridge University Press, 1993).

[34] L. Blanchet, T. Damour, B.R. Iyer, C.M. Will and A.G. Wiseman, Phys. Rev. Lett. 74, 3515, (1995).

[35] B. Bhawal and S.V. Dhurandhar, private communication.

[36] R. Balasubramanian, A. Gopakumar, B.R. Iyer and B.S. Sathyaprakash, in preparation.

[37] B.S. Sathyaprakash and S.V. Dhurandhar, J. Computational Phys., 109, 215 (1993).

TABLE I. The estimated value of the parameters and their errors ${ }_{\mathrm{e}} \lambda\left(\sigma_{\lambda}\right)$ for the Newtonian waveform. The actual values of the parameters taken were $\hat{\tau}_{0}=5558.0 \mathrm{~ms}$ and $\hat{t}_{a}=200.0 \mathrm{~ms}$. Except for the parameter $\mathcal{A}$ all values are quoted in ms.

\begin{tabular}{cccccc}
\hline \hline & $\rho=7.5$ & $\rho=10.0$ & $\rho=12.5$ & $\rho=15.0$ & $\rho=20.0$ \\
\hline & & & & & \\
$\mathrm{e} \tau_{0}\left(\sigma_{\tau_{0}}\right)$ & $5557.4(29.7)$ & $5557.3(15.6)$ & $5557.6(9.0)$ & $5557.7(6.2)$ & $5558.5(3.9)$ \\
$\mathrm{e} t_{a}\left(\sigma_{t_{a}}\right)$ & $200.6(29.4)$ & $200.7(15.5)$ & $200.4(8.9)$ & $200.3(6.2)$ & $199.5(3.9)$ \\
$\mathrm{e} t_{C}\left(\sigma_{t_{C}}\right)$ & $5758.0(0.30)$ & $5758.0(0.20)$ & $5758.0(0.10)$ & $5758.0(0.08)$ & $5758(0.02)$ \\
$\mathrm{e} \mathcal{A}\left(\sigma_{\mathcal{A}}\right)$ & $7.696(0.96)$ & $10.12(0.98)$ & $12.582(0.99)$ & $15.067(0.99)$ & $20.05(0.99)$ \\
\hline \hline
\end{tabular}

TABLE II. The estimated value of the parameters and the errors in their estimation $\lambda_{\mathrm{e}}\left(\sigma_{\lambda}\right)$ for the post-Newtonian waveform. The actual values of the parameters taken were $\tau_{0}=5558.0 \mathrm{~ms}$, $\tau_{1}=684.0 \mathrm{~ms}$ and $t_{a}=300.0 \mathrm{~ms}$ Except for the parameter $\mathcal{A}$ all values are quoted in ms.

\begin{tabular}{cccccc}
\hline \hline \multicolumn{1}{c}{$\rho=10.0$} & $\rho=15.0$ & $\rho=20.0$ & $\rho=25.0$ & $\rho=30.0$ \\
\hline & & & & & \\
$\mathrm{e} \tau_{0}\left(\sigma_{\tau_{0}}\right)$ & $5554.9(136.1)$ & $5555.9(64.8)$ & $5557.2(30.1)$ & $5556.7(19.3)$ & $5558.7(13.6)$ \\
$\mathrm{e} \tau_{1}\left(\sigma_{\tau_{1}}\right)$ & $685.55(65)$ & $685.2(32.6)$ & $684.6(16.4)$ & $684.8(10.5)$ & $683.6(7.2)$ \\
$\mathrm{e} t_{a}\left(\sigma_{t_{a}}\right)$ & $301.49(73.26)$ & $300.88(33.4)$ & $300.28(14.5)$ & $300.5(9.4)$ & $299.7(6.8)$ \\
$\mathrm{e}{ }^{t}\left(\sigma_{t_{C}}\right)$ & $6.542(0.54)$ & $6.542(0.30)$ & $6.542(0.17)$ & $6.542(0.10)$ & $6.542(0.06)$ \\
$\mathrm{e} \mathcal{A}\left(\sigma_{\mathcal{A}}\right)$ & $10.25(0.97)$ & $15.15(0.98)$ & $20.11(.99)$ & $20.1(0.99)$ & $30.1(0.99)$ \\
\hline \hline
\end{tabular}


FIG. 1. This figure illustrates the correlation function along the line of curvature as a function of $\tau_{0}$, the Newtonian chirp time, for the following three cases: (i) when the filter is placed on the manifold (dotted line), (ii) when the filter is the 'average' signal vector over the region (dashed line) and (iii) when the filter is chosen to be an appropriate linear combination of the previous two filters (solid line).

FIG. 2. Contour diagram of the ambiguity function for the second post-Newtonian case.

FIG. 3. Dependence of the errors in the estimation of the parameters of the post-Newtonian waveform on the upper cutoff frequency. The SNR is kept fixed at a value of 10 .

FIG. 4. Dependence of the errors in the estimation of the parameters of the post-Newtonian waveform on the total mass of the binary keeping the distance to the binary fixed. The waveforms are cutoff at frequencies corresponding to the onset of the plunge orbit and the SNR is normalized at a value of 10 for a $1.4 M_{\odot}-10 M_{\odot}$ binary.

FIG. 5. Dependence of the errors in the estimation of parameters of the Newtonian waveform i.e. $\left\{\sigma_{\tau_{0}}, \sigma_{t_{a}}, \sigma_{t_{C}}\right\}$ as a function of SNR. The solid line represents the analytically computed errors whereas the dotted line represents the errors obtained through Monte Carlo simulations.

FIG. 6. Distributions of the measured values of the parameters for the case of Newtonian signal. The total number of noise realizations is 5000 .

FIG. 7. Dependence of the errors in the estimation of parameters of the post-Newtonian waveform i.e. $\left\{\sigma_{\tau_{0}}, \sigma_{\tau_{1}}, \sigma_{t_{a}}, \sigma_{t_{C}}\right\}$ as a function of SNR. The solid line represents the analytically computed errors whereas the dotted line represents the errors obtained through Monte Carlo simulations.

FIG. 8. Distributions of the measured parameters of the post-Newtonian waveform at a SNR of 25. The number of noise realizations is 5000 .

FIG. 9. The histogram of random variable ${ }_{\mathrm{m}} \mathcal{A}$ at an SNR of 20 . The variance in this parameter is independent of the SNR and is approximately equal to unity.

FIG. 10. The correlation between $t_{a}$ and $\Phi$ is illustrated. The phase parameter simply follows the time of arrival parameter.

FIG. 11. Distributions for the measured parameters of the post-Newtonian waveform incorporating the effect of the upper cutoff. The errors in the determination of the parameters is much smaller in this case. The total number of noise realizations is 5000 .

FIG. 12. The dependence of the errors on the SNR $(\rho)$ when the upper cutoff is incorporated into the waveform 


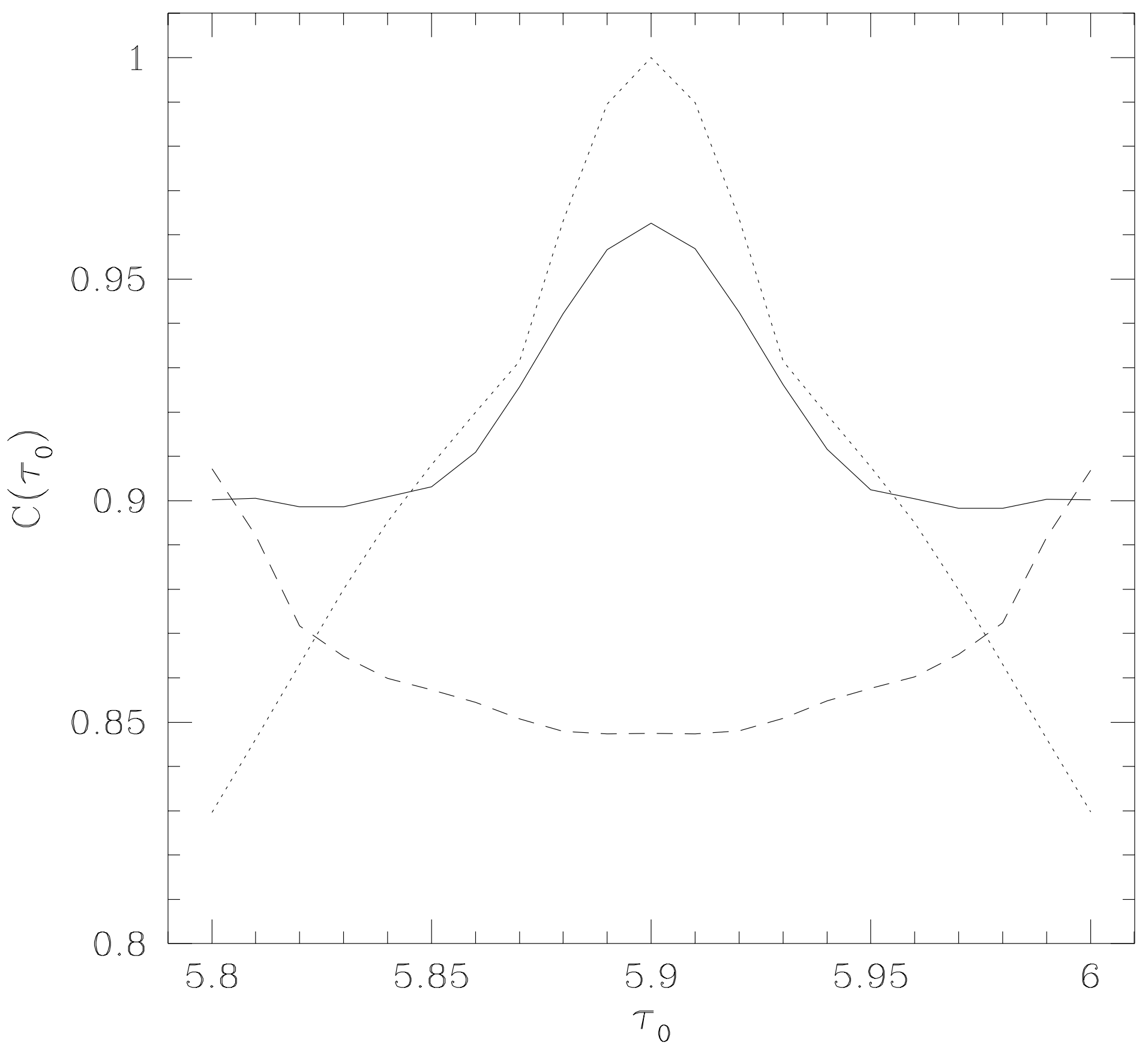




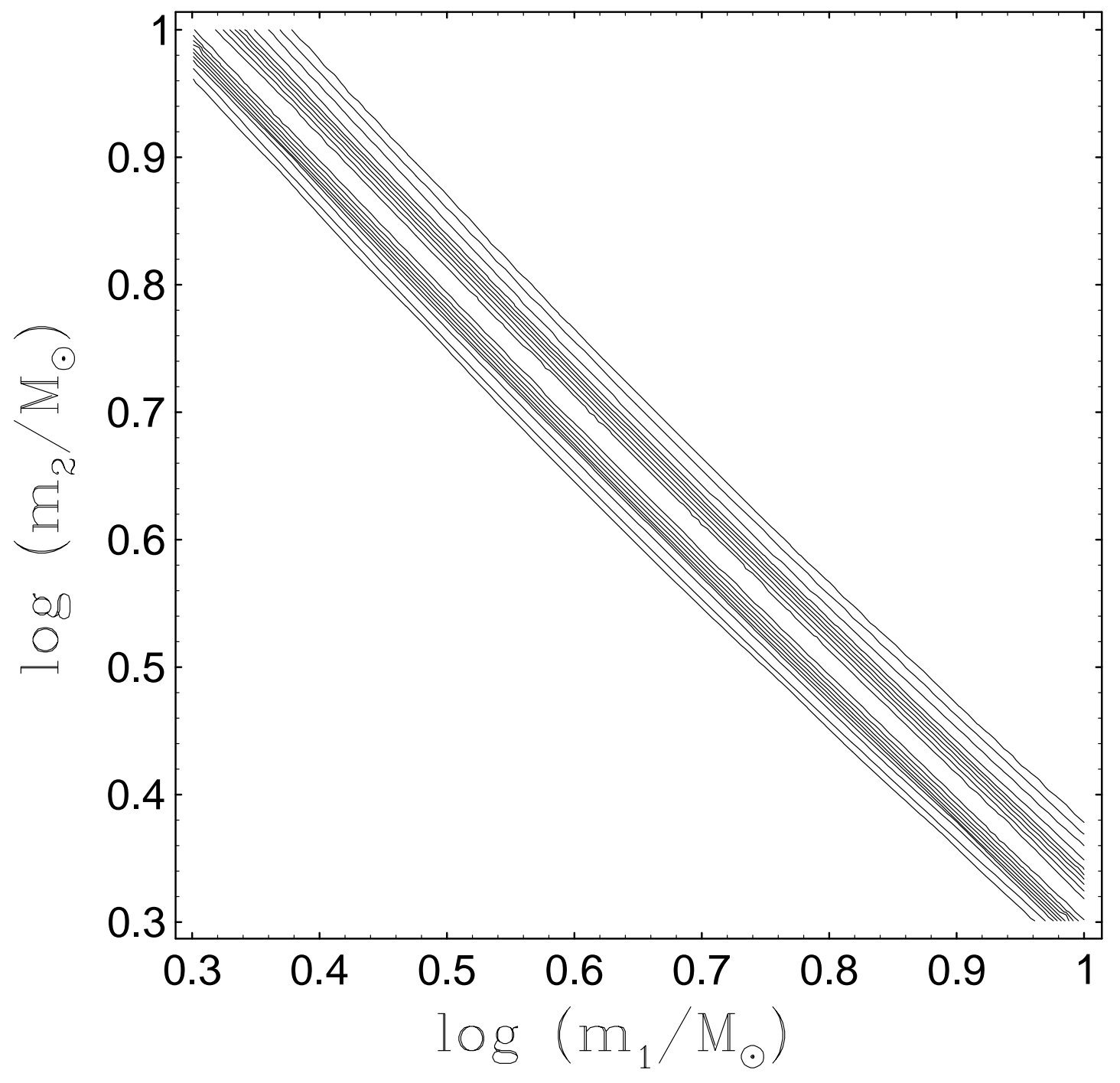




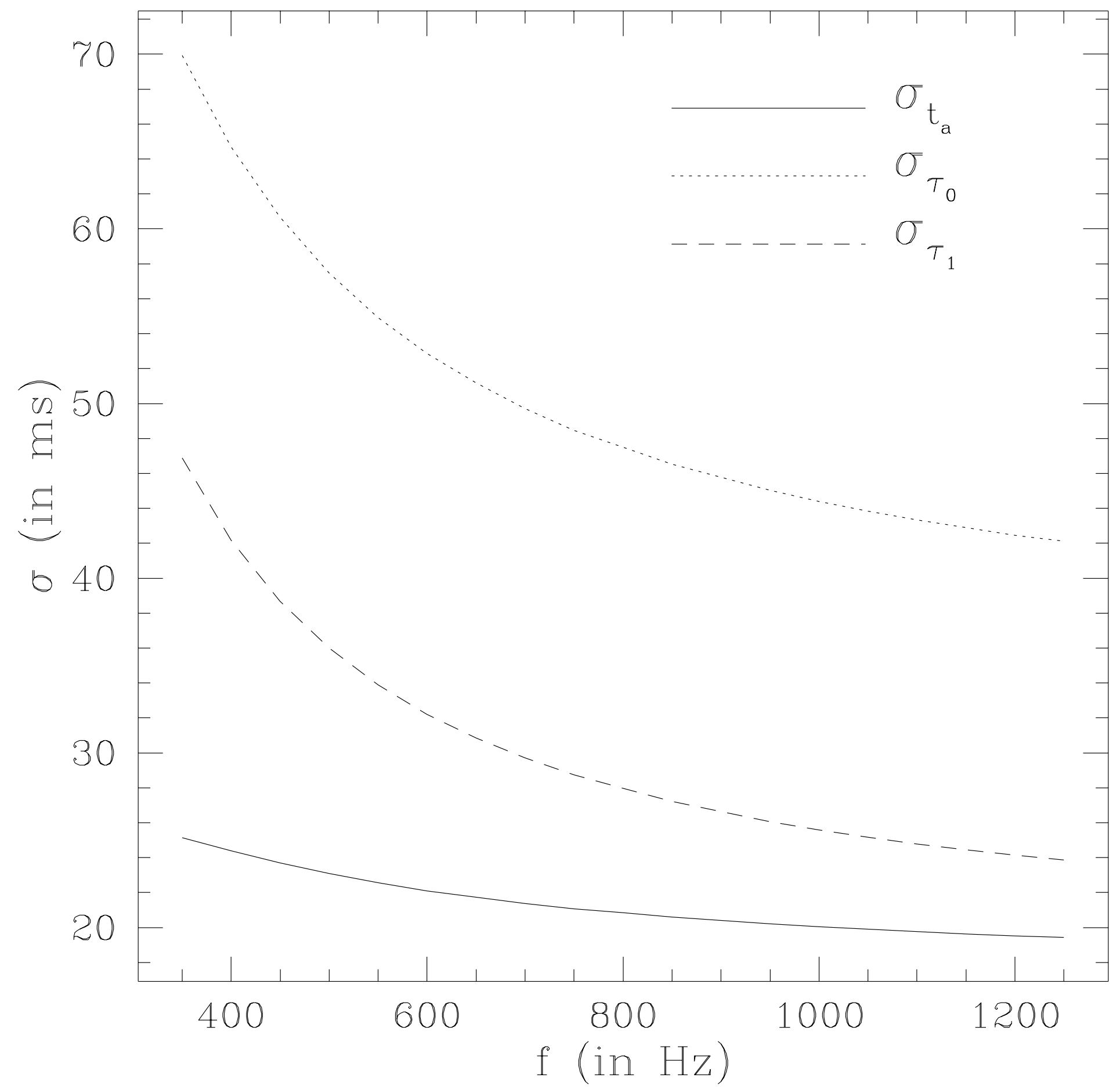




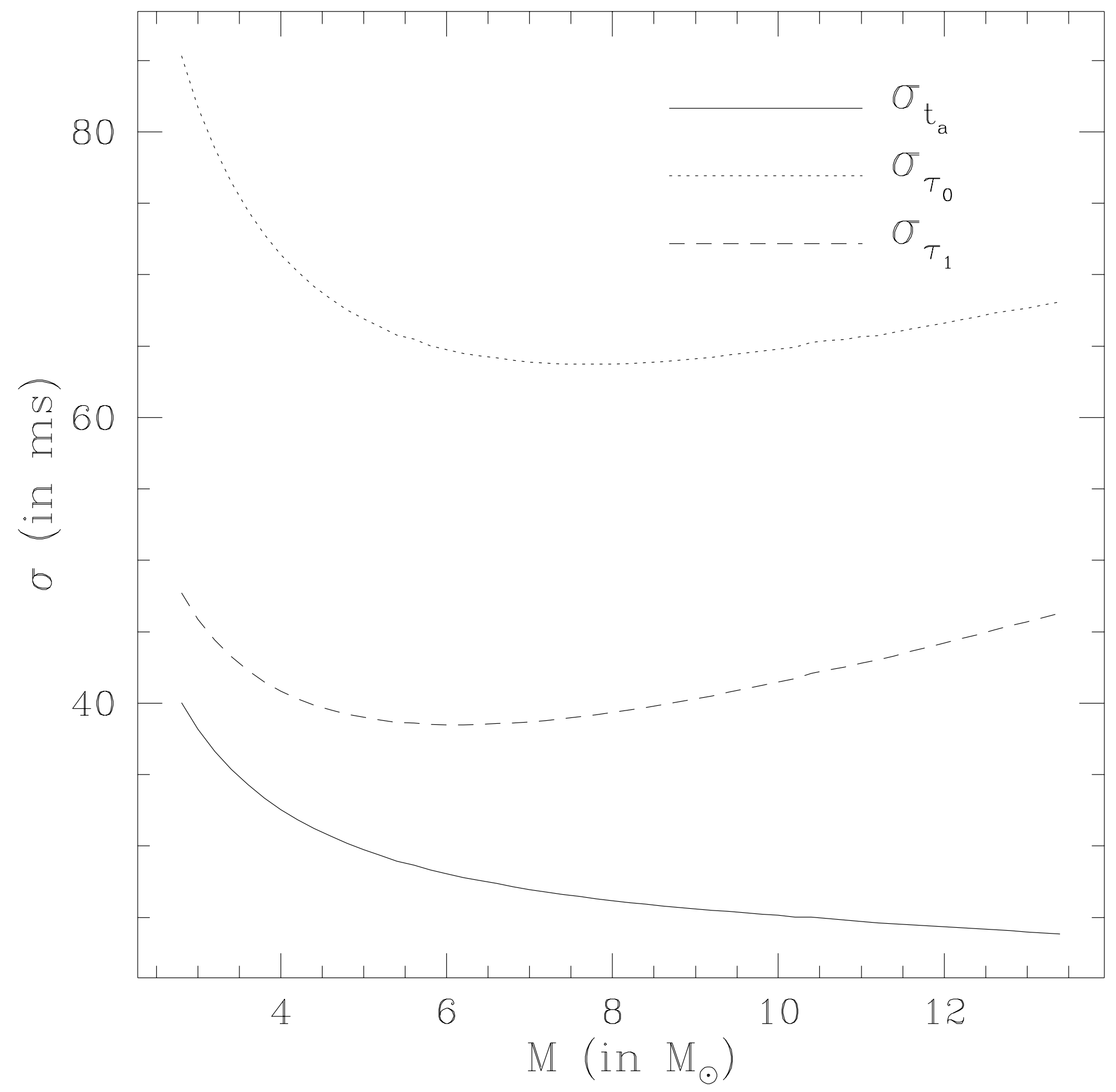




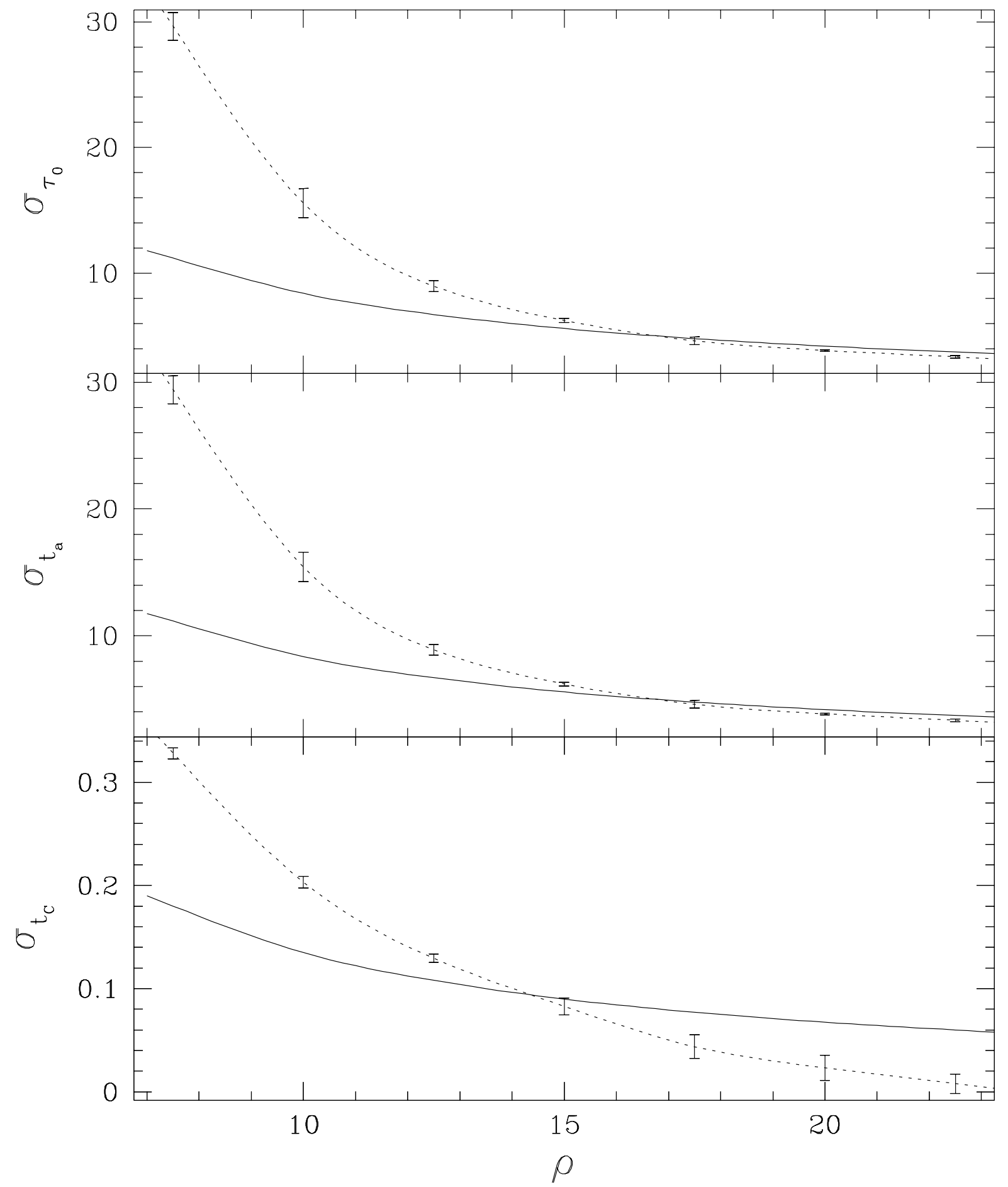



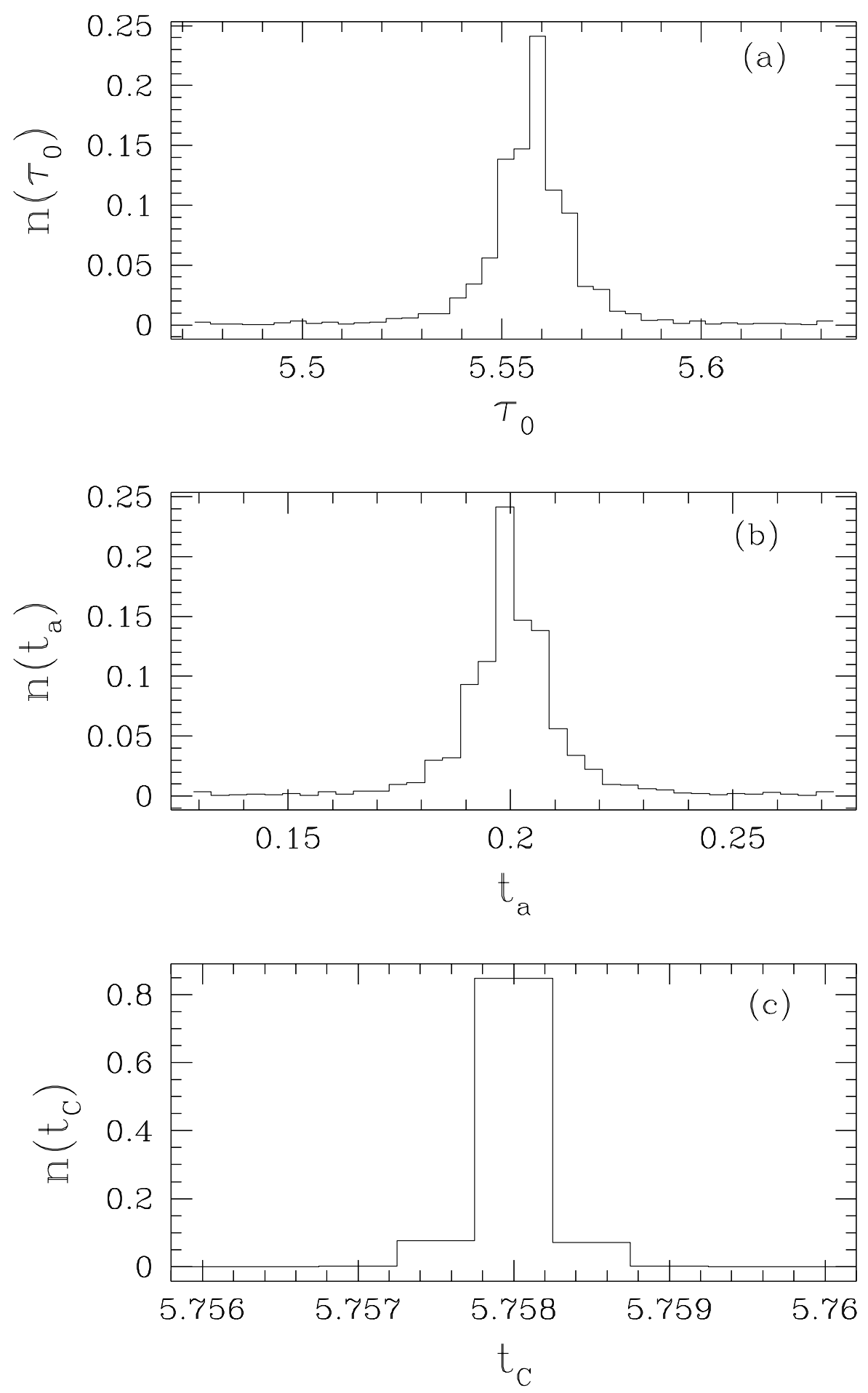

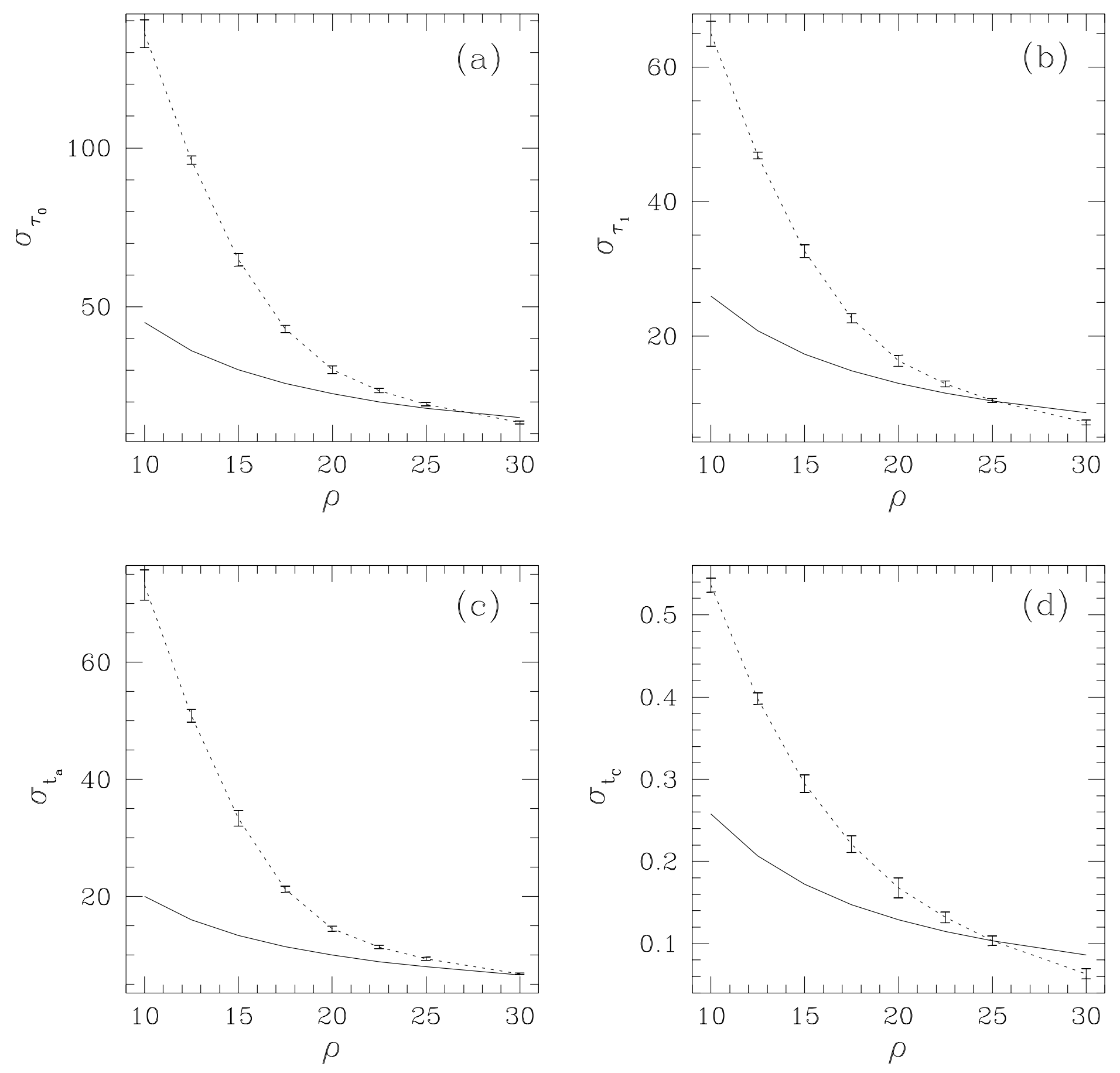

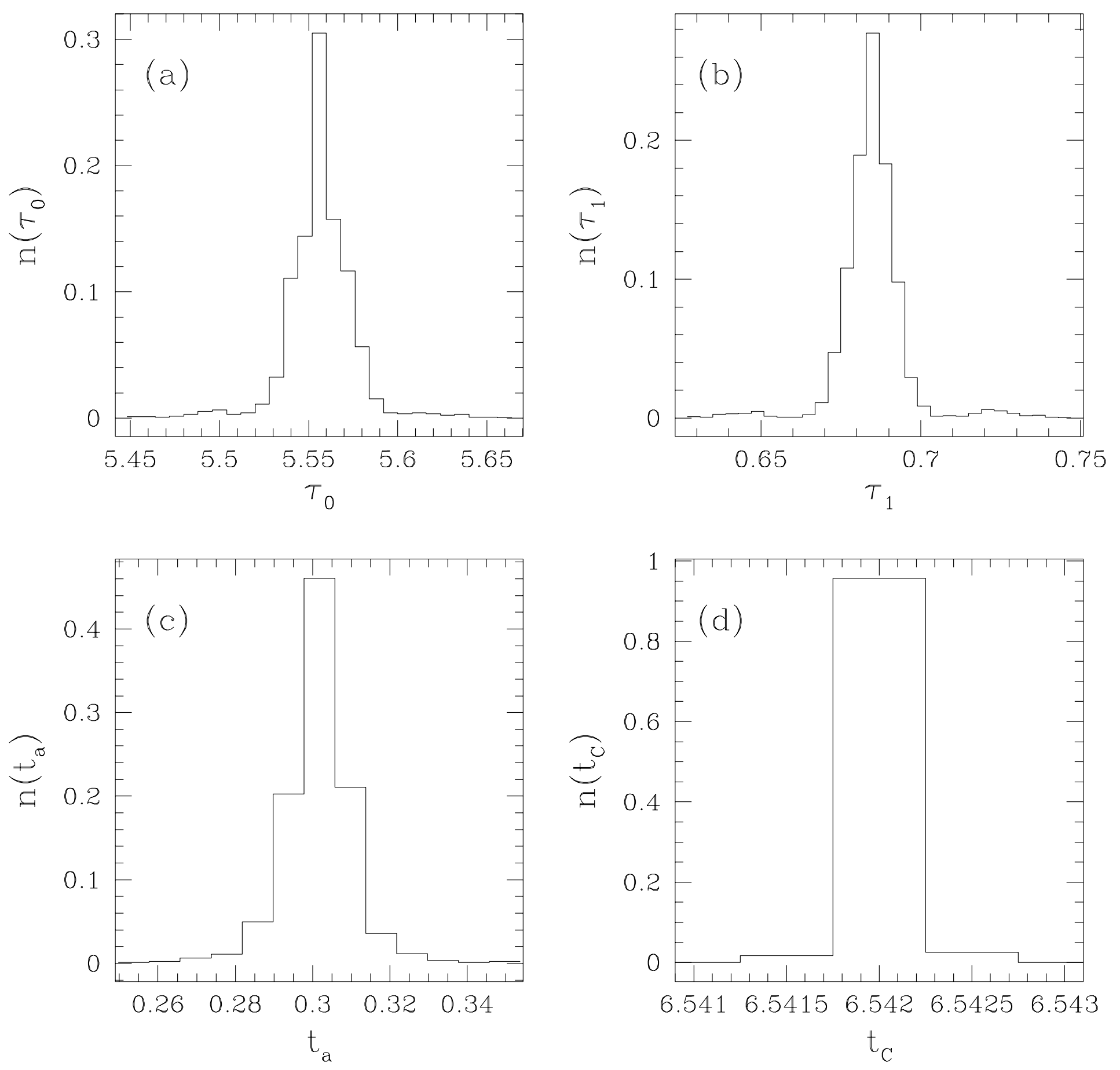


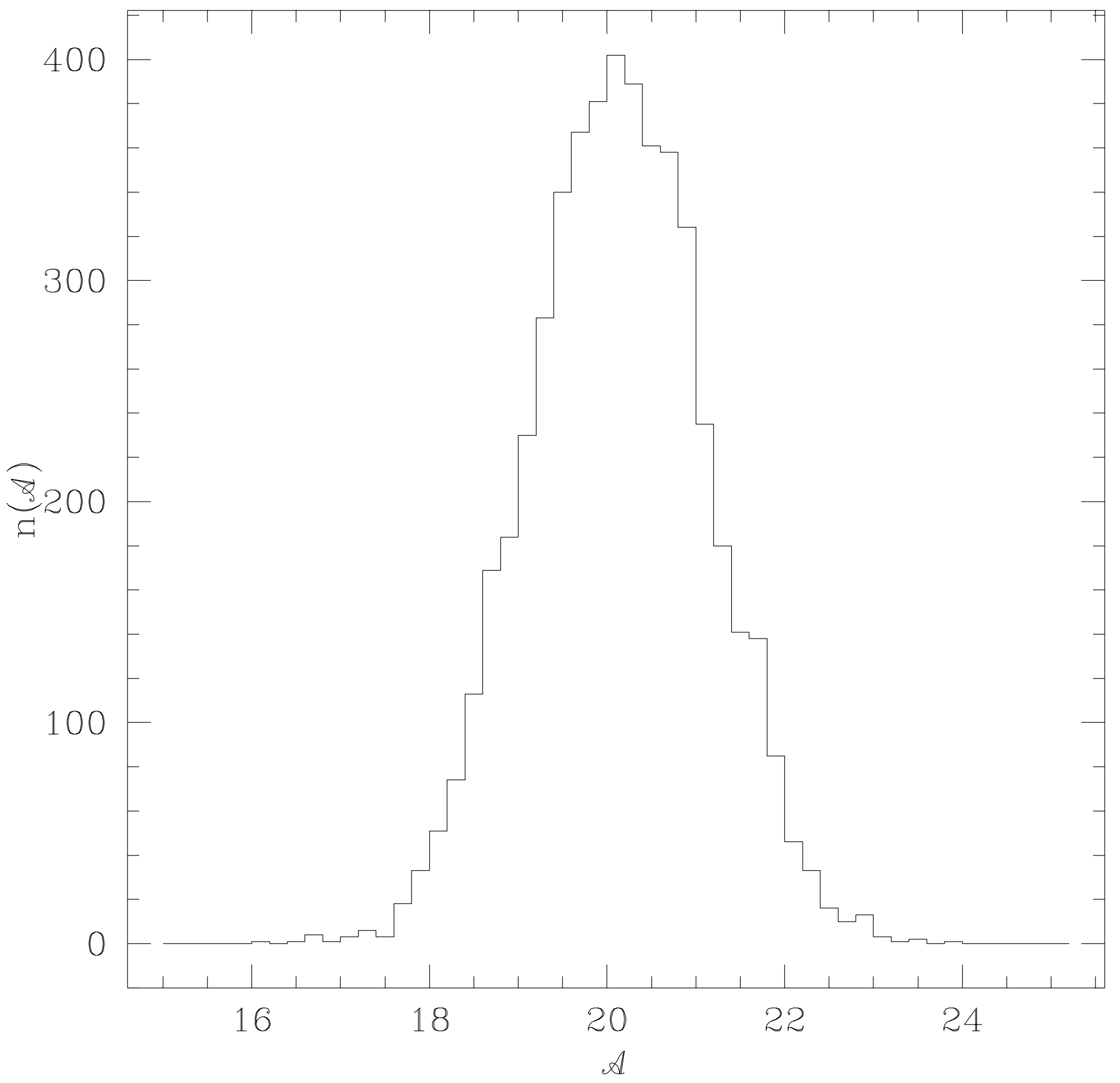




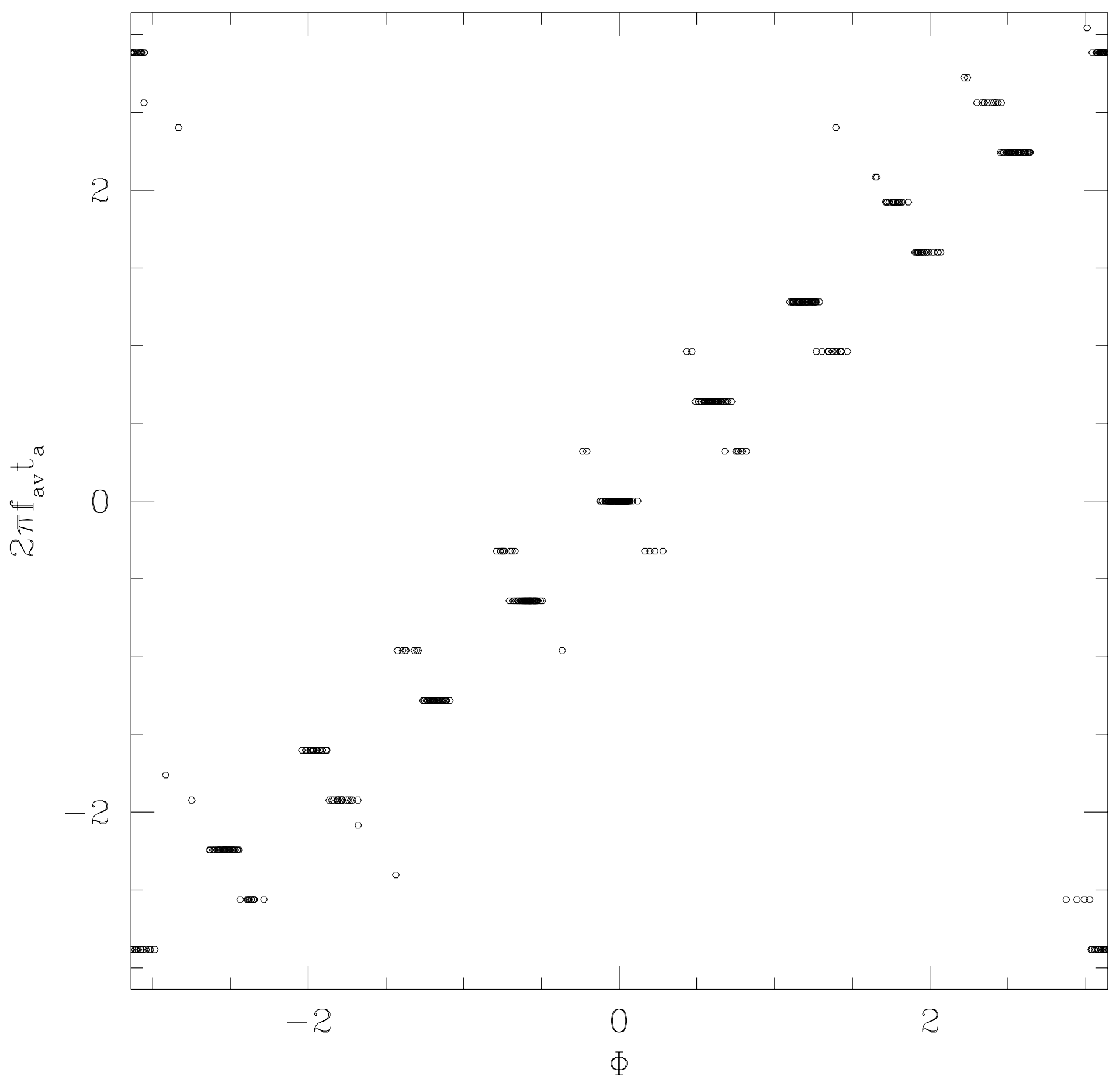



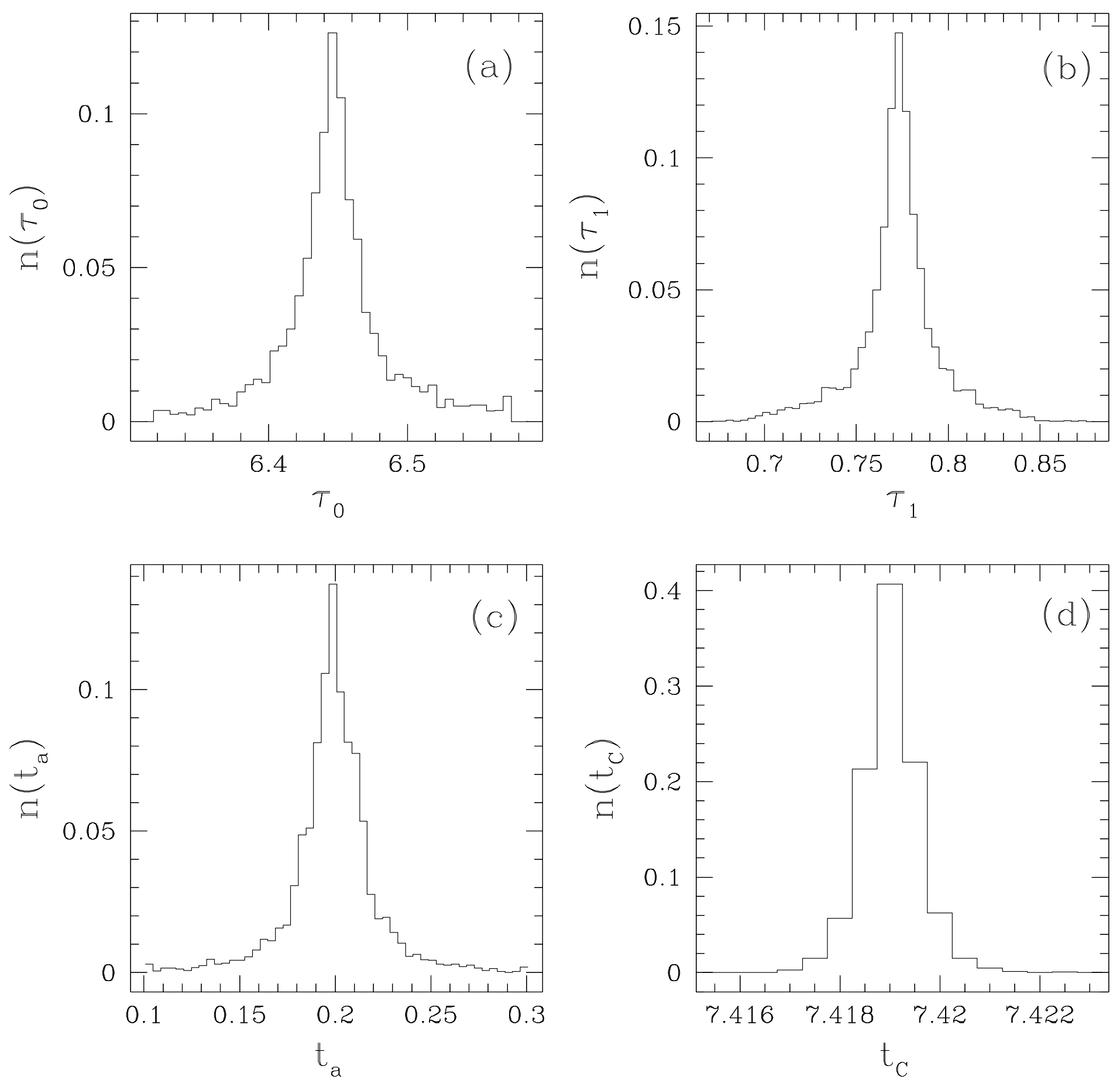

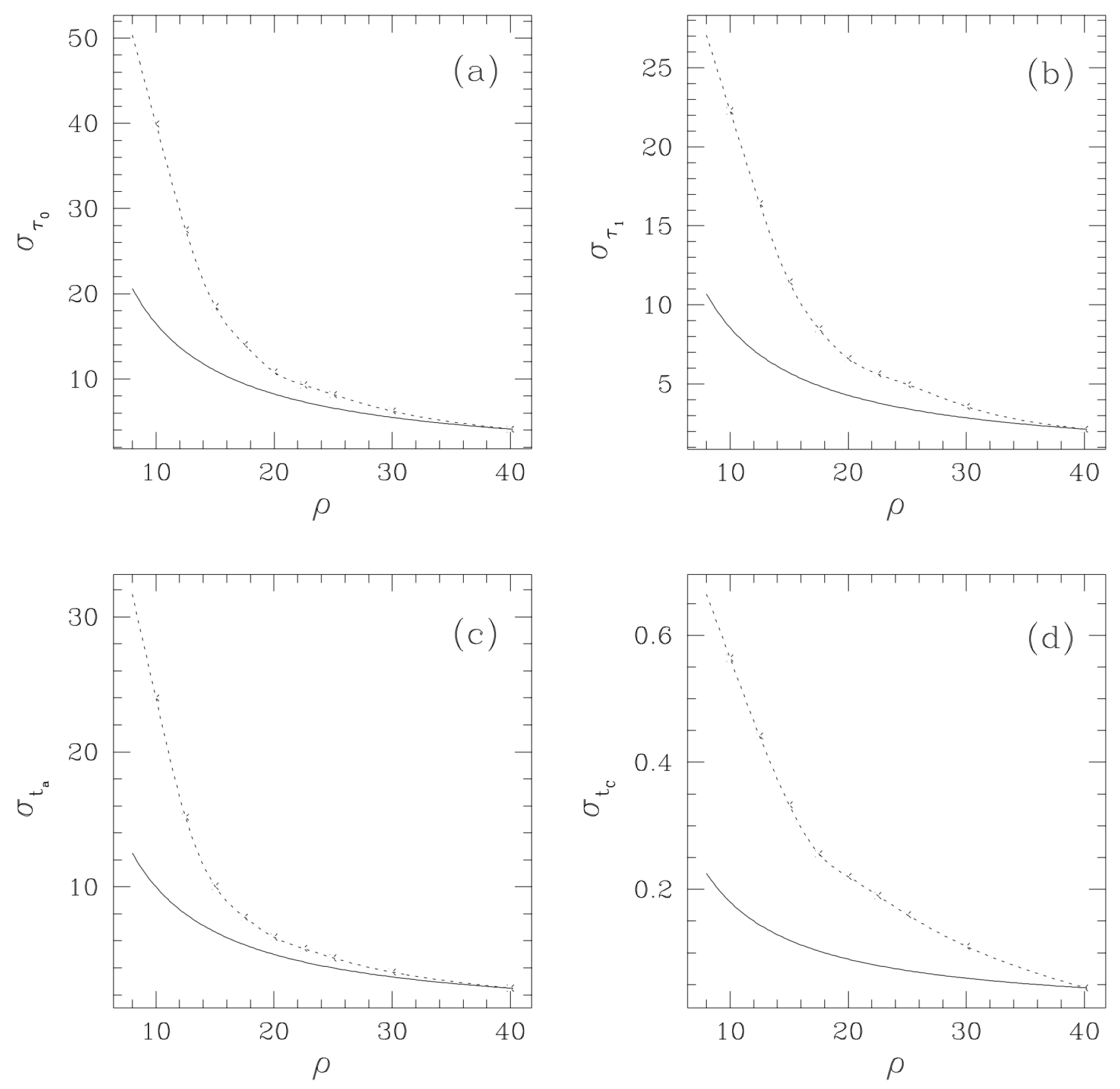